\title{
Non-smooth Mutants of Salmonella typhimurium: Differentiation by Phage Sensitivity and Genetic Mapping
}

\author{
By R. G. WILKINSON,* P. GEMSKI, Jun. AND B. A. D. STOCKER \\ Guinness-Lister Research Unit, Lister Institute of Preventive \\ Medicine, Chelsea Bridge Road, London, S.W.I
}

(Accepted for publication 20 December 197I)

\section{SUMMARY}

Non-smooth mutants of Salmonella typhimurium strain LT2 with known lipopolysaccharide (LPS) defects were tested for sensitivity to smooth-specific phages ( $P_{22}$ and $P_{22} h$ and a newly isolated phage, 9NA, active on $\mathrm{P}_{22}$ lysogens); Felix $\mathrm{O}$ phage; and several rough-specific phages including $\mathrm{C}_{2}$ I. Smooth strains were sensitive only to the smooth-specific and Felix $\mathrm{O}$ phages. Six $r f b$ mutants (unable to make $O$ chains) were sensitive to Felix $O$ and all the rough-specific phages except $C_{2} I$ (pattern R-sens). Of nine $r f a$ mutants (presumed to have LPS core defects) four were $\mathrm{R}$-sens, six were resistant to Felix $\mathrm{O}$ and some rough-specific phages (pattern $\mathrm{R}$-res-I), and one was also resistant to phage Br2 (pattern R-res-2). The phage sensitivity of phosphomannoisomerase (pmi) mutants was the same as that of $r f b$ mutants, except that they were partly sensitive to P22h. UDPgalactose-epimerasenegative mutants were sensitive to $\mathrm{C}_{2} \mathrm{I}$ and various rough-specific phages including $\mathrm{Br} 2$ (pattern Epi-I). An $r f c$ mutant (unable to polymerize $\mathrm{O}$ repeat units) was sensitive only to Felix $\mathrm{O}$ and $\mathrm{P}_{22}$ I (pattern Zsr). A part-rough mutant of class $\mathrm{D}$ (with abnormally few $\mathrm{O}$ chains) was incompletely resistant to smooth-specific phages, resistant to Felix $\mathrm{O}$ but sensitive to all rough-specific phages except $\mathrm{C}_{2}$ I (pattern D-I).

Spontaneous and mutagen-induced non-smooth mutants were isolated from LT2 strains with appropriate markers by selection with Felix $\mathrm{O}$ and/or $\mathrm{P} 22$ phage. (One parent strain used was non-lysogenic for Fels 2, for which LT2 wild-type is lysogenic. Lysogeny for Fels 2 did not affect sensitivity to the other phages.) Some mutants gave new sensitivity patterns. Mutants of these and of previously unmapped classes were crossed with smooth $\mathrm{Hfr}$ strains. The $r f_{c}$ loci of two mutants and pmi loci of two others were located in the gal-trp segment. Three mutants of pattern $\mathrm{R}$-sens yielded O-specific hapten but mapped near his; they are believed to be unable to transfer $\mathrm{O}$ chains from antigen carrier lipid to the LPS core as a result of mutation at $r f b T$. Six mutants of pattern R-sens were smooth in cultural and serological properties; they mapped near his and are probably leaky $r f b$ mutants. Many mutants had the class $D$ part-rough phenotype, divisible by phage sensitivities into patterns D-I, D-2 and D-3. Mutants of all three classes mapped near $x y l$; they are likely to be $r f a$ mutants, perhaps leaky, with LPS core defects which hinder but do not prevent attachment of $O$ chains. Two classes were sensitive to C2 I (Wilkinson \& Stocker, I968): $r f a H$ mutants, of pattern Epi-I, unable to add the main-chain galactose unit of the core; and $r f a G$ mutants, resistant to $\mathrm{Br} 2$ (pattern Epi-2), unable to add the proximal glucose unit. Both loci mapped in or near the strA-xyl-metA segment. Several non-smooth mutants did not grow in the presence of bile-salts. Three mutants $(r f a F)$ made LPS deficient of the distal heptose unit; one mutant ( $r f a E$ ) was unable to add the proximal heptose unit. Both these loci mapped in or near the strA-metA segment.

* Present address: School of Microbiology, University of Melbourne, Parkville, Victoria, Australia. 


\section{INTRODUCTION}

Rough $(R)$ variants of Enterobacteriaceae lack the $O$ (somatic) antigenic specificity of their smooth (wild-type) parents, in consequence of alteration in composition of the polysaccharide part of their somatic lipopolysaccharide (LPS). In smooth strains, but not their rough variants, the LPS core bears $O$ side-chains, each of which (in some or perhaps all $O$ groups) is a linear polymer of an O-specific oligosaccharide repeating unit whose composition varies between $O$ groups (for review of LPS chemistry see Lüderitz, Jann \& Wheat, I968). In salmonellas of group B the $O$ repeating unit contains galactose, mannose, rhamnose and abequose (usually acetylated) and (in some strains) glucose; the LPS of rough mutants lacks the ' $O$-specific' sugars, mannose, rhamnose and abequose. Correlated genetical and biochemical studies of a series of rough mutants of Salmonella typhimurium strain LT2 showed that they fell into two main groups (Beckmann, Subbaiah \& Stocker, 1964; Nikaido, Nikaido, Subbaiah \& Stocker, 1964; Subbaiah \& Stocker, 1964). One group, termed $r f b$ (originally $r o u B$ ) had mutations near his and were inferred to have defects in biosynthesis of the $\mathrm{O}$-specific side-chains. The other group, termed $r f a$ (formerly $\operatorname{rou} A$ ) had mutations which mapped in the $\operatorname{str} A-$ met $A$ region: they accumulated O-specific polysaccharide (hapten) but did not attach it to the LPS core, and were inferred to have defects in the assembly of the core. In addition to these two classes galactose-negative mutants which lack UDPgalactose-epimerase and therefore are unable to convert UDPglucose to UDPgalactose (the precursor of the galactose units of LPS) are phenotypically rough, unless provided with exogenous galactose (Fukasawa \& Nikaido, I960). Similarly, S. typhimurium mutants lacking phosphomannoisomerase cannot synthesize GDPmannose (unless provided with exogenous mannose) and are therefore unable to synthesize the mannose-containing $O$ repeat unit and so are phenotypically rough (Rosen et al. 1965). Some Salmonella mutants and inter-species hybrids are intermediate between smooth and rough, both in cultural and serological properties and in the amount of O-specific material in their LPS. Two phenotypic classes have been distinguished (Naide, Nikaido, Mäkelä, Wilkinson \& Stocker, 1965). Strains termed semirough (SR) are unable to polymerize $O$ repeating units and their LPS therefore contains side-chains each comprising only a single $O$ unit (Mäkelä I966; Nikaido, Naide \& Mäkelä, I966). The LPS of the other sort, now termed part-rough, contains some $\mathrm{O}$ polymer and it is thought that the reduction in content of $\mathrm{O}$-specific sugars results from reduction in the number of $O$ chains, instead of from reduction in their average length.

Burnet (1927) and others observed that some phages attack only smooth forms of a given species, whereas others attack only rough variants but not their smooth parents. Phage $\mathbf{P}_{22}$, used as a general transducing phage for Salmonella typhimurium, is smooth-specific, attacking only smooth strains possessing $O$ factor I 2 (Zinder, I953). Subbaiah \& Stocker (I964) noted some correlation of type of rough mutation with sensitivity, or resistance to certain roughspecific phages. We here describe an investigation of the activity of a more extensive set of smooth-specific and rough-specific phages (including a newly isolated smooth-specific phage unrelated to phage $\mathrm{P}_{22}$ ) on rough mutants of known types derived from S. typhimurium strain LT2 and their use for the classification of newly isolated rough mutants of this strain, most of them obtained by selection for phage resistance. (For other reports on use of these and other phages to differentiate rough mutants of Salmonella spp. see Schmidt \& Lïderitz, I969; Lindberg \& Hellerqvist, 197I.) The nature of the LPS defect in some of the new classes has been determined, either by us or by our biochemical collaborators. Genetical experiments have indicated the approximate map position of the loci affected in several of these new classes and in mutants of several previously known, but unmapped, classes. Two new classes, 


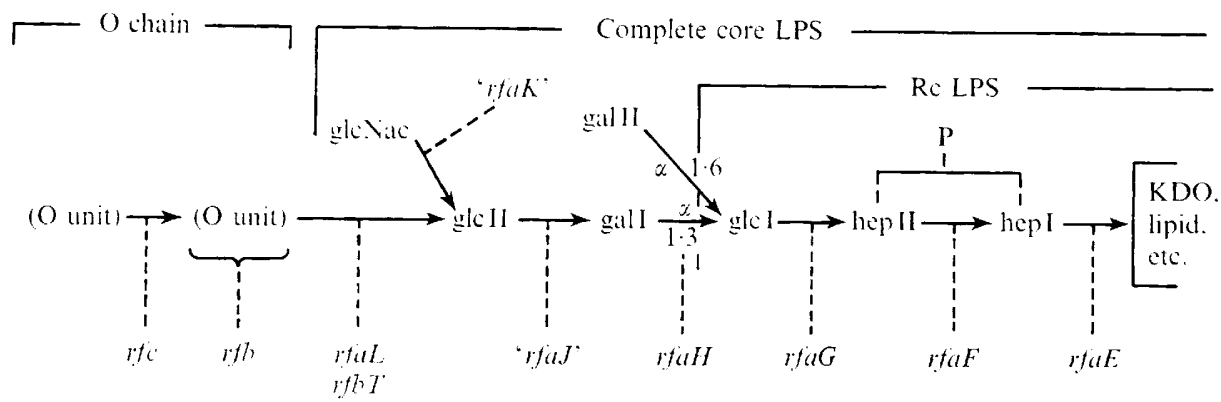

Fig. I. Structure of Salmonella lipopolysaccharide and symbols proposed for genes concerned with formation of its constituent units. gal $=$ Galactose; glc $=$ glucose; glcNac $=N$-acetylglucosamine; hep $=$ heptose; $K D O=2$-keto 3 -deoxyoctanoic acid, $\mathrm{P}=$ phosphate. The brace over the heptose units indicates that it is not known which is phosphorylated. In Salmonella typhimurium the $O$ repeating unit is:

$$
\begin{aligned}
& \text { abequose } \\
& \rightarrow \text { (mannose } \rightarrow \text { rhamnose } \rightarrow \text { galactose }) \rightarrow
\end{aligned}
$$

The abequose is acetylated in most strains, and the galactose sometimes bears a glucosyl sidebranch. Genes shown in quotation marks are postulated on theoretical grounds, although mutants with corresponding defects have not been unequivocally identified.

including one class in which hapten is accumulated, have been found to result from mutation near his, and several other new classes from mutation in the str-xyl-metA region. Two of the latter classes, discovered in the course of this investigation, are the $r f a G$ and $r f a H$ mutants, with defects affecting the activity of transferases for formation of, respectively, the proximal glucose and the main-chain galactose unit of the LPS core side-chain (Fig. I): as these mutants have been described (Osborn, I968; Wilkinson \& Stocker, 1968) we here mention them only briefly.

\section{METHODS}

\section{Bacterial strains}

The strains used (Tables I and 2) are all derivatives of Salmonella typhimurium strain LT2. Genotype symbols accord with the standard convention (Demerec, Adelberg, Clark \& Hartman, 1966). Mutations affecting synthesis of LPS core and O-specific side-chains are now designated, respectively, $r f a$ and $r f b$, instead of $r o u A$ and $r o u B$; mutants with defects in polymerization of $O$ repeat units are called $r f c$; and mutants with undetermined defects in LPS structure are indicated $r f$. The expression 'semirough' (SR) is now reserved for mutants with defects in polymerization of $\mathrm{O}$ repeat units: other phenotypes intermediate between smooth and complete rough are termed 'part-rough'. (See Mäkelä \& Stocker, I969, for a scheme of designation of loci concerned with LPS biosynthesis.)

\section{Phages}

Phages $P_{22}$ and $P_{22}$. We used the non-lysogenizing variants, P22.c2 (obtained from Dr M. Levine) and P22h.c2 (obtained from Dr T. F. Anderson). Phage P22 attacks only smooth (or 'partly smooth') lines of Salmonella typhimurium (Zinder, I953). Phage P22h is an extended host-range variant, active on a P22-resistant mutant of strain LT2 (Yamamoto \& Anderson, 196I), later found to be a part-rough mutant of class D (Naide et al. 1965). Though it attacks some part-rough mutants resistant to phage $\mathbf{P} 22$ it probably requires the presence of some O-specific material in LPS for adsorption (Gemski \& Stocker, 1967). 
Table I. Main strains used

Strain no. Genotype $\uparrow$

Reference and/or origin

(a) Smooth parents* of non-smooth mutants

SDI4 LT2 metAz2trpB2HI-bH2-e,n,x cured of Fels 2

\begin{tabular}{|c|c|}
\hline SL696\$ & as SDI 4 but also flaA66strAI20 \\
\hline SL697 & $\begin{array}{l}\text { as SDI4 but also flaA66strAI20 } \\
\left(\mathrm{P}_{22}\right)\end{array}$ \\
\hline SLIO27٪ & $\begin{array}{l}\text { as SDI4 but flaA66strAI20 } \\
x y l-404 \text { metE55I }\end{array}$ \\
\hline SL 1092 & $\begin{array}{l}\text { LT2 hisD27trpA8 (CoI EI-30)HI-a } \\
\quad H 2-e, n, x g a l-437 x y l-4 I 2\end{array}$ \\
\hline SLI093 & $\begin{array}{l}\text { as SDI } 4 \text { but also flaAstr } A x y \text { lmet } E \\
\text { malA } 49 I\end{array}$ \\
\hline SLIO94 & as SDI 4 but flaAstr AxylmetE ilv \\
\hline SL2 I 57 & $\begin{array}{l}\text { as SDI } 4 \text { but flaAstr Axylmet E } \\
\text { ilv ser }\end{array}$ \\
\hline SL2227 & LT2 pyrEI25xylrha \\
\hline \multicolumn{2}{|c|}{ b) Smooth donor strains } \\
\hline SR305 & LT2 his-23galK5omet $C_{3}$ HfrA \\
\hline su396 & LT2 proA26( $\mathrm{P} 22) \mathrm{HfrB2}$ \\
\hline SL862 & $\begin{array}{c}\text { LT2 hisD27trpA8 (CoIEI-30) } \\
\text { HI-aH2-e,n,xoafAmtl }\end{array}$ \\
\hline
\end{tabular}

Flagellar antigen genes transduced from Salmonella abony sw803 (Spicer \& Datta, 1959). For loss of prophage Fels 2, see Results section, also Yamamoto (1967) and Kuo \& Stocker (1970)

Joys \& Stocker (1965)

P22-lysogenic derivative of sL696

The originally undetected met $E$ mutation apparently resulted from the mutagen exposure used to obtain the $x y l$ mutation (Kuo \& Stocker, 1970)

Flagellar antigen genes transduced from $S$. abortusequi sL23. The gal mutation did not cause any alteration of phage sensitivity pattern

EMS-induced mal mutant of SLI027; mal-49I maps between $\operatorname{str} A$ and $x y l$

EMS-induced $i l v$ mutant of SLIO27

EMS-induced ser mutant of SLIO94

$x y l$ and $r h a$ Mutations induced by EMS treatment

Sanderson (1970)

Sanderson (1970)

* Some non-smooth mutants were isolated from strains SL90I and SL1030, which were $r f c$ and $p m i$, respectively (see Table 2 , sections $a$ and $b$ ).

$\uparrow$ For meaning of locus symbols see Demerec et al. (1966). Genotype designations curtailed except at first mention. EMS = ethyl methane sulphonate.

$\$$ sL 696 , the streptomycin-resistant parent of SL1027, the source of many of our rough mutants was obtained (S. M. Smith \& B. A. D. Stocker, unpublished) by the shaken-flask procedure (see Methods) and assumed to be a single-step mutant, strAr2o. It has since been found to be a double mutant, with one mutation to streptomycin resistance, permitting only slow growth in the presence of high concentration of streptomycin, and another 'modifier' mutation permitting rapid growth in such conditions. The modifier locus maps near $\operatorname{str} A$ but is not co-transduced with it by phage P22 (B. A. D. Stocker, T. Kuo \& J. Stern, unpublished). After the present work was completed it was also discovered (Kuo \& Stocker, 1970) that strain SLI027, a xylosenegative mutant obtained from a metA trpB parent by EMS treatment, was met $E^{-}$as well as $x y l^{-}$; because of the second block in methionine synthesis methionine-independent recombinants obtained from SLIO27 or its descendants must have obtained both met $^{+}$and $m e t E^{+}$from the donor - but these loci are close together (Fig. 2) so that the interpretation of our crosses is little affected.

\begin{tabular}{|c|c|}
\hline \multicolumn{2}{|c|}{ Mutant } \\
\hline $\begin{array}{c}\text { Strain } \\
\text { no. }\end{array}$ & $\begin{array}{c}\text { Mutation } \\
\text { no. }\end{array}$ \\
\hline
\end{tabular}

(a) Mutants giving pattern $\mathrm{Zsr}$

$\begin{array}{llll}\text { SL90I } & r f c-497 & \text { SL697 } & \text { Sp., reisolate } \\ \text { SLI034 } & r f c-465 & \text { SL696 } & \text { Sp., P22 }+ \text { Ffm }+ \text { Br6o }\end{array}$

(b) Phosphomannoisomerase-deficient

$\begin{array}{llll}\text { SLIO30 } & \text { pmi-404 } & \text { SLIO27 } & \text { EMS, mannose-negative } \\ \text { SLIIOI } & p m i-408 & \text { SL9OI } & \text { EMS, mannose-negative } \\ & & & \text { colony }\end{array}$

(c) Mutants giving pattern $\mathrm{R}$-sens, culturally rough, hapten-positive

$\begin{array}{lllll}\text { SLI196 } & r f b-580 & \text { SLI092 } & \text { EMS, P22 } & \text { Close to his } \\ \text { SLII97 } & r f b-604 & \text { SLI092 } & \text { EMS, P22 } & \text { Close to his } \\ \text { SLII98 } & r f b-605 & \text { SLI092 } & \text { EMS, P22 } & \text { Close to his }\end{array}$

Inferred map position§ of $r f$. (or pmi) mutation

Between gal and trp

Between gal and trp (Table 4)

Probably between $g a l$ and $f a A$, linked to $g a l$

Linked to $g a l$ and $r f c$

Close to his 
Table 2 cont.

$\overbrace{\substack{\text { Strain } \\ \text { no. }}}^{\text {Mutant }} \underbrace{\text { Mutal }}_{\begin{array}{c}\text { Mutation } \\ \text { no. }\end{array}}$

$\overbrace{\text { Parent } \dagger}^{\text {Origin }} \overbrace{\text { Method }}^{+}$

Inferred map position§ of
$r f$. (or pmi) mutation

(d) Mutants giving pattern R-sens, but culturally smooth

$\begin{array}{lllll}\text { SLIII } & r f b-548 & \text { SLI092 } & \text { EMS, P22 } & \text { Close to his } \\ \text { SLIII3 } & r f b-598 & \text { SL1092 } & \text { EMS, P22 } & \text { Close to his } \\ \text { SLIII4 } & r f b-599 & \text { SLI092 } & \text { EMS, P22 } & \text { Close to his } \\ \text { SLIII5 } & r f b-600 & \text { SLI092 } & \text { EMS, P22 } & \text { Close to his } \\ \text { SLIII6 } & r f b-601 & \text { SLI092 } & \text { EMS, P22 } & \text { Close to his } \\ \text { SLIII7 } & r f b-602 & \text { SLI092 } & \text { EMS, P22 } & \text { Close to } h i s\end{array}$

(e) Mutants giving pattern D-I

\begin{tabular}{|c|c|c|c|c|}
\hline SLIO35 & $r f a-469$ & SL696 & Sp., P22 & Linked to met $A$ and $\operatorname{str} A$ \\
\hline SLIO36 & $r f a-474$ & SLIO27 & $\mathrm{Sp} ., \mathrm{P}_{22}+\mathrm{FO}$ & $\begin{array}{l}\text { Between } \operatorname{str} A \text { and metE, } \\
\text { close to } x y l\end{array}$ \\
\hline SL1054 & $r f a-486$ & SLIO30 & $\begin{array}{l}\text { Sp., P22 + FO } \\
\text { (with mannose) }\end{array}$ & $\begin{array}{l}\text { Between str } A \text { and metE, } \\
\text { close to } x y l\end{array}$ \\
\hline SL2IO5 & $r f a-940$ & SU396 & Sp., $\mathrm{P}_{22}+\mathrm{FO}$ & Close to $x y l$ \\
\hline SL2 I 74 & $r f a-80 I$ & SLIO93 & Sp., $P_{22}$ & $\begin{array}{l}\text { Between mal } A \text { and met } E \text {, } \\
\text { close to } x y l\end{array}$ \\
\hline SL2 178 & $r f a-800$ & SLIO93 & $\mathrm{Sp} ., \mathrm{P}_{22}+\mathrm{FO}$ & $\begin{array}{l}\text { Between malA and } m e t E \text {, } \\
\text { close to } x y l\end{array}$ \\
\hline SL2I 79 & $r f a-938$ & SL2 I 57 & $\mathrm{Sp} ., \mathrm{P}_{22}+\mathrm{FO}$ & Close to $x y l$ \\
\hline SL2 I 80 & $r f a-939$ & SL2 I 57 & Sp., $\mathrm{P}_{22}+\mathrm{FO}$ & Close to $x y l$ \\
\hline SL2IO5 & $r f a-940$ & SL2227 & Sp., $\mathrm{P}_{22}+\mathrm{FO}$ & Between $x y l$ and $p y r E$ \\
\hline \multicolumn{5}{|c|}{ f) Mutants giving pattern D-2 } \\
\hline SLI I IO & $r f a-490$ & SLIO30 & $\begin{array}{l}\text { Sp., } \mathrm{P} 22+\mathrm{FO}+6 \mathrm{SR} \\
\text { (with mannose) }\end{array}$ & $\begin{array}{l}\text { Between } \operatorname{str} A \text { and } m e t E \text {, } \\
\quad \text { close to } x y l\end{array}$ \\
\hline SL I I I I & $r f a-527$ & SLIO27 & EMS, $P_{22}$ & $\begin{array}{l}\text { Between str } A \text { and met } E \text {, } \\
\text { close to } x y l\end{array}$ \\
\hline \multicolumn{3}{|l|}{ ) Mutan } & $\mathrm{Sp} ., \mathrm{P}_{22}+\mathrm{FO}$ & Close to $x y l$ \\
\hline
\end{tabular}

(h) Mutant giving pattern Hep-I

$\begin{array}{llll}\text { SLIIO2 } & \text { SL } 102743 & \text { EMS, P22 }+ \text { FO } & \text { In or near str } A-m e t A \text { segment }\end{array}$

(i) Mutants giving pattern Hep-2

$\begin{array}{lllll}\text { SLI I 8I } & r f a-5 I I & \text { SLI027 } & \text { Sp., FO } & \text { In or near str } A-\text { met } A \text { segment } \\ \text { SLI I 82 } & r f a-537 & \text { SLI027 } & \text { EMS, FO } & \text { In or near str } A-m e t A \text { segment } \\ \text { SLI I 83 } & r f a-546 & \text { SLI027 } & \text { EMS, FO+Ffm } & \text { In or near str } A-\text { met } A \text { segment }\end{array}$

* For genetically analysed C2I-sensitive mutants, of patterns Epi-I (class $r f a H$ ) and Epi-2 (class $r f a G$ ), see Wilkinson \& Stocker ( 1968 ).

$\dagger$ See Table I for genotypes, etc. of smooth parent strains, and sections $a$ and $b$ of this table for details of non-smooth parent strains, i.e. SL9OI and SLIO3O.

$\ddagger \mathrm{Sp} .=$ mutants isolated from untreated cultures; $\mathrm{EMS}=$ mutants isolated from ethyl methane sulphonate-treated cultures; P22, FO, etc. = phages used for selection (the $c 2$ variant was used in case of phage P22); 'with mannose' = selection of phage-resistant mutants of pmi parents on mannose-supplemented medium. (Mannose-non-fermenting mutants were detected as pale colonies on MacConkey-type medium.)

$\S$ For details of Hfr donors used see text and Table $\mathrm{I} b$.

If Most smooth recombinants from crosses of $\mathrm{P}_{22}$ lysogenic HfrB2 donor with class D part-rough mutants were P22-lysogenic presumably because they had adsorbed phage P22 particles liberated by donor cells. Some non-smooth recombinants also were P22-lysogenic, as shown by their resistance to phage P22 I.c2 and confirmed by demonstration of release of phage $\mathbf{P} 22$. Presumably these non-smooth recombinants also had been infected by free phage P22; though class D mutants behave as resistant to phage $P_{22}$ in our pattern tests, they are susceptible to transduction by this phage (Gemski \& Stocker, 1967) and must therefore retain some ability to adsorb it. 
Phage $9 N A$. This newly isolated smooth-specific phage, unrelated to phage $\mathrm{P}_{22}$, is described under Results.

Felix anti-O phage. This virulent phage (hereinafter termed Felix $\mathrm{O}$ phage) is active on smooth forms of Salmonella typhimurium (and of most other Salmonella species (Kallings, 1967)) and also on some rough variants (Felix \& Callow, 1943; Callow, I959; Subbaiah \& Stocker, 1964). The stock we used, originally obtained from Dr E. S. Anderson, derived from Felix's anti-O phage no. 2 (Felix \& Callow, 1943); it is reputedly indistinguishable from Felix's international standard 'anti-O' phage (Fildes \& Kay, 1955). This phage is morphologically similar and serologically related to phage C2I (A. M. Lawn \& R. G. Wilkinson, in preparation).

Phages $6 S R, \phi X-174, S_{13}$ and $B r 2$. Phage 6SR, also known as A59, anti-R and $\phi R$ (Fildes, I954; Kay, I955, I962; Tucker, I96I) was obtained from Dr E. S. Anderson. It was isolated by Felix in 1953 (Kay, 1962) and described as an 'anti-rough' phage. Phage $\phi \mathrm{X}-\mathrm{I} 74$ was isolated by Sertic \& Boulgakov (1935) as a phage active on a rough strain of Salmonella typhi and assigned to their antigenic group ten. Phage $\mathrm{SI}_{3}$ was isolated by Burnet (I927, 1929). We obtained these two phages from Dr N. Mendelson. Phages $\phi \mathrm{X}-\mathrm{I} 74$ and SI 3 are serologically and genetically related (Zahler, 1958; Tessman \& Shleser, 1963) and contain single-stranded DNA (Sinsheimer, I959; Tessman, 1959). Phage 6SR is believed also to be a single-stranded DNA phage (Tucker, 196I; Kay, 1962). In our experiments, phages $\phi \mathrm{X}-\mathrm{I} 74$ and $\mathrm{S}$ I 3 attacked the same range of non-smooth mutants of strain LT2. Phage Br2, our designation for a phage received from Dr H. Brandis and described by him as phage 2 (Brand is, 1956, 1966), attacked only rough derivatives of strain LT2, including some which were resistant to phages 6SR, $\phi \mathrm{X}-\mathrm{I} 74$ and SI3. However an anti-6SR serum also neutralized phages $\phi \mathrm{X}-\mathrm{I} 74, \mathrm{SI}_{3}$ and $\mathrm{Br}$, at different rates (A. M. Lawn \& R. G. Wilkinson, in preparation).

Phages Ffm and Br6o. These phages, received from Dr H. Brandis as phages Ffm and 60 (Brandis, 1956, 1966) attacked most of our rough derivatives of strain LT2, but not the smooth parent strains. These two phages are morphologically similar (A. M. Lawn, personal communication) and serologically related (H. Brandis, personal communication; R. G. Wilkinson, unpublished observations).

Phage C21. This phage (obtained from Dr S. Brenner), was isolated by Burnet \& McKie (1933). It attacks some wild-type Escherichia coli strains (e.g. strain B) but in the case of KI 2 attacks only mutants unable to make UDPgalactose, through absence of UDPgalactoseepimerase or of UDPglucose pyrophosphorylase (Shedlovsky \& Brenner, 1963). It is likewise inactive on Salmonella typhimurium strain LT2 wild-type but active on its mutants unable to synthesize UDPgalactose, and is also active on certain $r f a$ mutants, deficient in transferases for formation of the proximal glucose or the proximal galactose units of the LPS core sidechains (Osborn, 1968; Wilkinson \& Stocker, I968). It is morphologically similar and serologically related to Felix $\mathbf{O}$ phage, though different from it in host-range.

Phage P221. We used a non-lysogenizing variant, P221.c2, obtained from Dr T.F. Anderson. This phage was originally found in stocks of phage $\mathrm{P}_{22}$ grown in certain propagating strains. It differs from phage P22 in its serological character, morphology and hostrange (Yamamoto \& Anderson, 196I) and has been shown to be a recombinant of phage $\mathrm{P}_{22}$ and an unrelated phage carried by the propagating strains concerned (Yamamoto \& Weir, I966). 


\section{Media}

The complete media used were 'Oxoid' blood agar base (code CM55) and 'Oxoid' nutrient broth (code CMI). The defined medium used was Davis Minimal (Lederberg, 1950), citrate being omitted for tests on utilization of mannose, etc. Semisolid nutrient gelatin agar was used for testing motility. Sugar fermentation was tested on a MacConkey-type indicator medium without lactose and with $0.5 \%$ of the appropriate sugar added. Streptomycin ( $\mathrm{I} \mathrm{mg} / \mathrm{ml}$ ) was used for selection of streptomycin-resistant recombinants and for scoring streptomycin-resistance of recombinants.

\section{Genetical methods}

Isolation of mutants. Ethyl methane sulphonate (EMS) was used as a mutagen (Loveless \& Howarth, 1959). A broth culture, grown overnight at $37^{\circ}$ without aeration, was diluted tenfold into a solution of EMS, $4.4 \%(\mathrm{v} / \mathrm{v})$, in minimal salts solution: after 20 min at $37^{\circ}$ samples of suitable dilutions were spread on nutrient agar for selection of phage-resistant mutants, or on MacConkey-type indicator plates for isolation of non-fermenting mutants. Phage-resistant mutants were isolated from growth (separate colonies or confluent growth) developing in areas of lysis produced by application of drops of a phage (or mixture of phages), at $10^{8}$ plaque-forming units (p.f.u.)/ml, to nutrient agar plates surface-inoculated with untreated or mutagen-treated broth cultures of bacteria. To obtain mutants able to grow well in the presence of even high concentrations of streptomycin, a broth culture of a sensitive strain was shaken overnight in a partly-filled flask at $37^{\circ}$, an equal volume of broth + streptomycin ( $\mathrm{I} \mathrm{mg} / \mathrm{ml}$ ), was added and the flask shaken for a further $24 \mathrm{~h}$, then streaked on nutrient agar + streptomycin (to give final concentration of $\mathrm{I} \mathrm{mg} / \mathrm{ml}$ ). (See footnote to Table I for str genotype of SLI027, the parent of many non-smooth mutants, and derived from a streptomycin-resistant mutant obtained by this procedure.)

$H f r$ crosses. Log-phase broth cultures of donor and recipient, 0.2 and $\mathrm{I} \cdot 0 \mathrm{ml}$ respectively, were inoculated together into $50 \mathrm{ml}$ volumes of broth at $37^{\circ}$. After $3 \mathrm{~h}$ at $37^{\circ}$ unshaken, samples were centrifuged, washed once and resuspended at $\times$ Io concentration in distilled water: $0.1 \mathrm{ml}$ samples of this suspension and various dilutions were spread on plates of selective media. After one or two days of incubation, recombinant colonies were picked from the selective medium and purified by single-colony re-isolation on the same medium, then scored for segregating characters, usually by replication (using a multi-prong replicator) to appropriate media. In some cases recombinants were replicated with a velvet pad directly from selective media. Sensitivity to phages was determined by applying a droplet of phage ( $10^{8}$ p.f.u. $/ \mathrm{ml}$ ) peripherally to a dried-in drop of culture on the surface of a nutrient agar plate. After $5 \mathrm{~h}$ incubation, the crescent-shaped areas of partly lysed growth produced by sensitive strains were easily distinguished from the circular patches of growth of phageresistant strains. Sensitivity to appropriate phages proved a reliable and convenient method of determining the smooth or rough character of recombinants. In interpreting the results of $\mathrm{Hfr}$ crosses, the representation of an unselected donor allele in half or more of the recombinants from a given selection was taken as indicating fairly close linkage of the unselected locus to the selected donor locus, because in Salmonella $\mathrm{Hfr}$ crosses (in contrast to the situation in Escherichia coli) unselected donor loci not closely linked to the selected donor locus, even if injected earlier than it, are represented in much less than $50 \%$ of recombinants. When several loci of known map position were segregating, the numbers of additional crossovers required to account for the observed distribution of recombinant classes was calculated for each possible position of the locus to be mapped; this method of analysis is illustrated in Table 4 . 


\section{Serological methods}

The presence of $O$ antigens was tested by slide agglutination with an anti-4,5,12 serum (obtained from the Serological Standards Laboratory, Central Public Health Laboratory, Colindale), diluted fourfold in $0.2 \% \mathrm{NaCl}$ (to avoid saline agglutination of rough strains). To test for presence of $\mathrm{O}$-specific hapten a dense washed-cell suspension (from a mass culture, grown on 11 of nutrient agar contained in a tray) was mixed with an equal volume of I $N$ cold trichloracetic acid: the mixture was stirred at $4^{\circ}$ for $2 \mathrm{~h}$. After centrifugation in the cold 5 volumes of acetone were added to the supernatant, and the resulting flocculent precipitate allowed to settle, washed once in cold acetone and dried over $\mathrm{P}_{2} \mathrm{O}_{5}$. This material, dissolved in saline, was tested by ring precipitation test in capillary tubes with a rabbit anti-4, 5,I2 precipitating serum, obtained from Dr A. M. Staub.

\section{Phage methods}

The phages mentioned above were initially re-isolated from single plaques, then propagated on suitable LT2 lines (non-lysogenic for Fels 2, carried by LT2 wild-type-see Results) by the soft agar layer method. Lysates were sterilized by chloroform treatment or by Seitz filtration and titrated by the 'drop-on-lawn' method (Gemski \& Stocker, 1967) or in soft agar layers. For phage sensitivity tests nutrient agar plates were surface-inoculated by flooding from broth cultures, allowed to dry and then spotted with drops or loopful amounts of phage, usually at $10^{8}$ p.f.u. $/ \mathrm{ml}$.

\section{RESULTS}

\section{Lysogeny of LT2}

Most of the non-smooth mutants to be described were isolated from derivatives of strain SDI4 of Spicer \& Datta (1959), which is Salmonella typhimurium LT2 metA22 trpB2 given the flagellar antigen alleles $H I-b$ and $H 2$-enx of $S$. abony by two successive tranductions using phage P22. In the course of crosses of SDI4 derivatives with other LT2 lines it was found that SDI4 was sensitive to a temperate phage carried by LT2 wild-type (B. A. D. Stocker \& S. M. Smith, unpublished data). Strain SRI20, described as LT2 cured of a B phage (Zinder, I958), was likewise sensitive to a phage carried by strain LT2 wild-type, but apparently absent from strain SDI4. As the phage concerned is heat-labile it falls into group B of Boyd (I950). Tests on a set of lysogenic derivatives of strain QI of Boyd, each carrying a different B phage, showed that the LT2 phage missing from the LT2 derivatives SDI4 and SRI 20 was indistiguishable, by the criterion of reciprocal lysogenic immunity, from a phage termed B8 (J. S. K. Boyd, personal communication). Yamamoto (1967) has shown that strain LT2 wild-type is lysogenic for two phages active on Boyd's indicator strain QI, which he terms Fels I and Fels 2. Strains SDI4 and SRI20 do not carry Fels 2 (N. Yamamoto, personal communication). Lysogeny or non-lysogeny for Fels 2 did not appear to affect sensitivity to any of the phages used in the recognition and differentiation of non-smooth mutants; these phages were, however, always propagated on derivatives of strain SDI4, so as to avoid their contamination with phage Fels 2.

\section{Isolation of a new smooth-specific phage, 9 NA}

A newly isolated phage, termed 9NA, is described because it proved of value in differentiating smooth and non-smooth strains. Soft streptomycin-nutrient-agar layer plates were inoculated with London sewage filtered through paper, together with a streptomycinresistant smooth Salmonella typhimurium strain. A phage derived from a plaque on such a 
plate, purified by two successive single-plaque isolations, was designated 9 NA. Smooth LT2 strains, including those lysogenic for P22, were lysed by phage 9NA, whereas (non-leaky) rough mutants were resistant. The host range of $9 \mathrm{NA}$, so far as tested, resembles that of $\mathrm{P}_{22}$ and the other phages of group AI-A2 (Zinder, I953; Boyd \& Bidwell, I957); some Salmonella strains of species other than S. typhimurium, in groups B or D, were sensitive, whereas the few Escherichia coli strains tested were resistant. No LT2 strains lysogenic for 9NA were obtained in several trials, so presumably the phage is non-temperate. Attempts to transduce $p y r E$ and his with 9 NA were unsuccessful. Electron microscopy (R. G. Wilkinson \& A. M. Lawn, in preparation) showed $9 \mathrm{NA}$ to be a long-tailed phage, somewhat similar to phage P221, and quite unlike phage P22. Phage 9NA contains double-stranded DNA, of mean GC content about $40 \%$ (Brown, 1970).

\section{Phage patterns of P22-resistant mutants of known types}

Smooth, P22-sensitive Salmonella typhimurium LT2 strains, and mutants of various classes known to be resistant to P22, were tested for sensitivity to the phages listed in Table 3 (and, later, for bile-salt sensitivity - all grew well on nutrient agar + deoxycholate). With some exceptions, all mutants of a given class showed the same combination of sensitivities to some phages, resistance to others (Table 3). As two different kinds of biochemical defect might cause the same phage-resistance pattern it is convenient to assign names to some of the patterns.

Wild-type (smooth) pattern. Smooth strains were sensitive to phage P22, known to be a 'smooth-specific' phage (Zinder, 1953), and its extended host-range mutant, P22h, and to the unrelated smooth-specific phage $9 \mathrm{NA}$; they were sensitive also to Felix $\mathrm{O}$ phage, which is known to attack nearly all smooth salmonellas, whatever their O group (Kallings, 1967). They were resistant to all the other phages, which we therefore term 'rough-specific'. Smooth strains lysogenic for $\mathrm{P}_{22}$ were immune to $\mathrm{P}_{22}$ and $\mathrm{P}_{22} \mathrm{~h}$, but still sensitive to 9NA and Felix O phages.

$r f b$ Mutants. The six rouB (now termed $r f b$ ) mutants of Subbaiah \& Stocker (1964) were resistant to the three smooth-specific phages, sensitive to Felix $O$ and to all the roughspecific phages except $\mathrm{C}_{2} \mathrm{I}$. We term this the rough-sensitive pattern, or, for short, R-sens. One previously noted deviation (Subbaiah \& Stocker, 1964) from this pattern was the resistance to P221.c2 of TVI57, an rfb mutant which is P22-lysogenic; Yamamoto \& Anderson (I96I) noted that prophage P22 confers immunity to at least the original type of phage P22I.

Phosphomannoisomerase mutants. Mutants lacking phosphomannoisomerase, termed pmi, are unable to synthesize GDPmannose, the precursor of the mannose of the $O$ repeat unit, and are therefore phenotypically rough and $\mathrm{P} 22$-resistant when grown on ordinary media, making LPS lacking O chains, like that of $r f b$ mutants (Rosen et al. 1965). They become phenotypically smooth and P22-sensitive when their metabolic block is circumvented by the provision of exogenous mannose in the growth medium. We tested the pmi mutant M2 (Rosen et al. 1965) and three others, EPI, EP9 and EPI3, supplied by Dr M. J. Osborn. As expected, all four gave the smooth phage pattern on nutrient agar supplemented with mannose. On nutrient agar without mannose, they gave a characteristic pattern termed Pmi, identical with the R-sens pattern except that the pmi mutants were lysed by $\mathrm{P} 22 \mathrm{~h}$ and showed partial sensitivity to phage $\mathrm{P} 22$. The presence of glucose $(0.5 \%)$ in the agar prevented this latter sensitivity, but left the other reactions unaltered. Phage P22h is thought to attack only strains able to make at least some O-specific polysaccharide (Gemski \& Stocker, I967); if so, each of the pmi mutants must have been making some O polymer, therefore some GDP- 
Table 3. Sensitivity patterns of smooth and non-smooth derivatives of Salmonella typhimurium strain LT2

Lysis by phages*

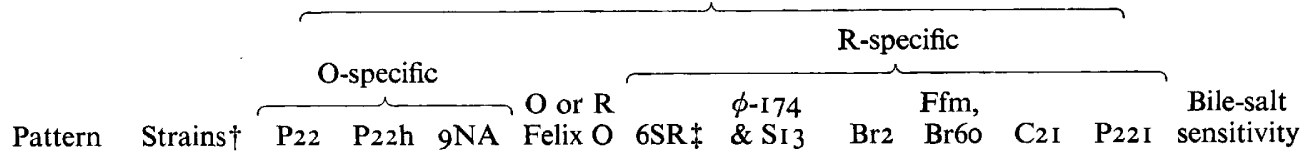

(a) Smooth strains

Smooth several $+++\quad+\quad-\quad-\quad-\quad-\quad-\quad-\quad$ res.

Smooth, several - $-+t_{-}-{ }_{-}-{ }_{-}$res.

P22-lyso-

genic

(b) Non-smooth mutants of previously described classes

\begin{tabular}{|c|c|c|c|c|c|c|c|c|c|c|c|c|}
\hline R-sens & $\begin{array}{l}6 r f b \text { and } \\
4 r f a \\
\text { mutants }\end{array}$ & - & - & - & + & + & + & + & + & - & + & res. \\
\hline Pmi & $\begin{array}{l}4 \text { pmi } \\
\text { mutants }\end{array}$ & \pm & + & - & + & + & + & + & + & - & + & res. \\
\hline R-res-I & $\begin{array}{l}7 r f a \\
\text { mutants }\end{array}$ & - & - & - & - & - & $t$ & + & + & - & + & res. \\
\hline R-res-2 & $\begin{array}{l}\text { I } r f a \\
\text { mutant }\end{array}$ & - & - & - & - & - & - & - & + & - & + & res. \\
\hline Epi-1 & $\begin{array}{l}5 \text { galE } \\
\text { mutants }\end{array}$ & - & - & - & - & - & - & + & + & + & + & res \\
\hline Zsr & $\begin{array}{l}\text { I } r f c \\
\text { mutant }\end{array}$ & - & - & - & + & - & - & - & - & - & + & res. \\
\hline D-I & $\begin{array}{l}\text { I class } D \\
\text { part-rough } \\
\text { mutant }\end{array}$ & - & \pm & - & - & + & + & + & + & - & + & res. \\
\hline
\end{tabular}

(c) Newly isolated non-smooth mutants defining new patterns

\begin{tabular}{|c|c|c|c|c|c|c|c|c|c|c|c|c|}
\hline D-2 & $\begin{array}{l}\text { Io class D } \\
\text { part-rough } \\
\text { mutants }\end{array}$ & \pm & \pm & \pm & - & - & - & + & + & - & + & res. \\
\hline D-3 & $\begin{array}{l}\text { I class } D \\
\text { part-rough } \\
\text { mutant }\end{array}$ & \pm & \pm & \pm & - & - & - & - & + & - & + & res. \\
\hline Hep-I & $\begin{array}{l}\text { I } r f a E \\
\text { mutant }\end{array}$ & - & - & - & - & - & - & - & \pm & - & \pm & sens. \\
\hline Hep-2 & $\begin{array}{l}3 r f a F \\
\text { mutants }\end{array}$ & - & - & - & - & - & - & - & + & - & + & sens. \\
\hline Epi-2 & $\begin{array}{l}8 r f a G \\
\text { mutants }\end{array}$ & - & - & - & - & - & - & - & + & + & + & res. \\
\hline
\end{tabular}

* Effect of phage at about $10^{8}$ plaque-forming units $/ \mathrm{ml}$ applied to surface-inoculated nutrient agar plate; $+=$ confluent lysis, $-=$ no effect, $\pm=$ partial sensitivity ('thinning' on plaques) and/or variable result.

+ The strains mentioned in part $(b)$ of this table are previously reported mutants mentioned in the text whose examination led to recognition of the patterns; additional mutants giving these patterns were encountered amongst newly isolated mutants (see text).

$\ddagger$ Though in the present investigation phage 6SR lysed only the strains indicated it has subsequently been observed to produce plaques on additional classes, including mutants giving patterns R-res-I, R-res-2 and Epi-I (Stocker, I969; A. Lindberg, personal communication). 
mannose, presumably because the pmi block was leaky, or because there is some unknown, relatively inefficient biosynthetic route to GDPmannose. The pmi mutants were tested for ability to ferment mannose and to utilize it as sole carbon source. Mutant EP9 though scored as non-fermenting on a mannose indicator plate produced acid in mannose peptone water and grew moderately well on mannose minimal medium without citrate; the other three mutants by these tests appeared non-leaky.

$r f a$ Mutants. Four of the original twelve rouA (now termed $r f a$ ) mutants were noted (Subbaiah \& Stocker, I964) to differ from the rest (and to resemble $r f b$ mutants) by their sensitivity to phages Felix $\mathrm{O}$ and $6 \mathrm{SR}$. We found these four mutants gave the complete $\mathrm{R}$-sens pattern. The other eight $r f a$ mutants were all resistant to the smooth-specific phages and also to Felix O and 6SR (but see Stocker (1969) and footnote to Table 3 for variation in reactions with phage $6 \mathrm{SR}$ ). They were all sensitive to the rough-specific phages $\mathrm{Br} 60$, Ffm and $\mathrm{P}_{22 \mathrm{I}}$ but resistant to $\mathrm{C}_{21}$. The remaining rough-specific phages $\mathrm{Br} 2, \phi \mathrm{X}-\mathrm{I} 74$ and $\mathrm{SI}_{3}$, attacked seven $r f a$ mutants, pattern R-res-I, but did not affect the other $r f a$ mutant, TVI48, pattern R-res-2.

UDPgalactose-epimerase mutants. The galactose units of LPS core and O repeat unit are derived from UDPgalactose, which in the absence of exogenous galactose is made from UDPglucose by the action of UDPgalactose-4-epimerase. Mutants lacking this enzyme, termed galE, are in consequence phenotypically rough and resistant to phage P22 when grown without exogenous galactose. The conversion of exogenous galactose to UDPgalactose involves the action of galactose kinase and of galactose phosphate uridyl transferase, determined respectively by genes galK and galT in the same operon as galE. Four mutants lacking only epimerase were available, i.e. strain LT2-MI of Fukasawa \& Nikaido (1960) and three mutants isolated in this laboratory (S. M. Smith \& B. A. D. Stocker, unpublished). All four mutants on nutrient agar gave the same pattern, Epi-I ; they were resistant to smooth-specific phages and to Felix $\mathrm{O}$ phage, resistant to the rough-specific phages 6SR, $\phi \mathrm{X}-\mathrm{I} 74$ and $\mathrm{SI}_{3}$, but sensitive to $\mathrm{Ffm}, \mathrm{Br} 60$ and $\mathrm{Br}$, and also to $\mathrm{C}_{2} \mathrm{I}$, a phage which attacks Escherichia coli KI2 mutants unable to synthesize UDPglucose or UDPgalactose but not their wild-type parents (Shedlovsky \& Brenner, 1963). Salmonella typhimurium mutants lacking only epimerase if grown with galactose can make UDPgalactose and so become phenotypically smooth and sensitive to phage P22 (Fukasawa \& Nikaido, 1960). We tested the phage sensitivity of our four mutants on nutrient agar supplemented with galactose $(0.5 \%$ ) (and also with glucose, at the same concentration, to prevent the growth-inhibitory or lytic effect of galactose on some epimerase mutants). The patterns obtained were the same as those of the galactose-positive smooth or rough parents. A mutant of $S$. typhimurium LT7, gal-5, in which all three enzymes were deficient, perhaps as a result of an operator mutation, remained P22-resistant even when grown with galactose (Fukasawa \& Nikaido, 1961). We confirmed this result and found that LT7 gal-5 gave the Epi-I phage pattern both on nutrient agar and on galactose-supplemented agar. Two LT2 mutants isolated in this laboratory resembled gal-5 by the absence (or very low levels) of all three enzymes (S. M. Smith \& B. A. D. Stocker, unpublished; T. Fukasawa, personal communication to B. A. D. Stocker). These two mutants showed the Epi-I pattern on unsupplemented medium, but on sugar-supplemented medium showed the parental phage pattern (smooth in one case, R-sens in the other). Several mutants lacking galactose-kinase gave, as expected, the smooth phage pattern.

$O$-polymerase ( $r f c$ or SR) mutants. Strain SL428, received from Dr Norton Zinder under the label SR 25 and described as a 'partly rough' P22-resistant mutant of an LT2 line cured of a $\mathrm{B}$ phage, was sensitive to phages Felix $\mathrm{O}$ and $\mathrm{P} 22 \mathrm{I}$ only. The same phage pattern was shown 
by strain SL9oI, a non-lysogenic P22-resistant but O-agglutinable variant isolated by $\mathrm{S}$. $\mathrm{M}$. Smith in this laboratory from a smooth P22-lysogenic LT2 line. The distinctive phage pattern of these two mutants was termed 'Zpr' (Stocker, 1969) because it was characteristic of Zinder's 'part-rough' mutant, but we shall hereinafter call it Zsr, because it is characteristic of the SR phenotype. Certain recombinants from crosses of group B with group C salmonellas also manifest the Zsr pattern, and resemble the two LT2 mutants also by cultural properties and LPS composition intermediate between those of smooth and rough strains (Mäkelä, 1966). This 'semirough' or SR phenotype results from inability to polymerize $O$ repeat units (Naide et al. 1965; Nikaido et al. 1966; Yuasa, Nakane \& Nikaido, 1970) resulting from mutation or loss by intergroup recombination of a polymerase gene (or genes) called $r f c$ (formerly rouC).

Part-rough mutants of class D. Yamamoto \& Anderson (196I) isolated from strain LT2 a P22-resistant mutant, termed st/22; it proved to be partly sensitive to an extended-host-range mutant of P22, termed P22h, and sensitive to phage P22 I, which did not attack the parent strain. Subbaiah \& Stocker (I964) found that phage P22 I . c2 attacked $r f a$ and $r f b$ derivatives of strain LT2, but not their smooth parents. We found that strain st/22 (SL733 in our collection) gave a distinctive phage pattern which we call D-I (formerly stt-I), comprising sensitivity to all the rough-specific phages except $\mathrm{C} 2 \mathrm{I}$, resistance to Felix $\mathrm{O}$ and partial sensitivity to one or more of the smooth-specific phages. This pattern of sensitivity suggests that both smooth and rough phage receptor sites are exposed in LPS. Strain St/22 (= SL733) proved to be intermediate between smooth and rough both in cultural and serological properties and in LPS sugar composition (Naide et al. 1965), but differed from strains of semirough (SR) phenotype (described above) by its phage sensitivity. Such strains were therefore termed 'part-rough, class $\mathrm{D}$ '. We show below that they are probably $r f a$ mutants with LPS defects which hinder but do not totally prevent attachment of $O$ chains to the LPS core, in some cases at least in consequence of 'leaky' mutations at rfa loci.

\section{Isolation, characterization and mapping of non-smooth mutants}

Spontaneous and mutagen-induced phage-resistant mutants were isolated from various genetically marked smooth LT2 lines by streaking out colonies from areas of lysis caused by application of drops of Felix O phage, alone or mixed with P22.c2, to surface-inoculated plates. Cultures which had been exposed to ethyl methane sulphonate produced many more colonies in the area of complete lysis caused by Felix $\mathrm{O}$ phage than did untreated control cultures. As mutants of patterns $\mathrm{R}$-sens and Zsr are sensitive to Felix $\mathrm{O}$ phage we attempted to isolate these classes from mutagen-treated cultures, by selection with phage P22.c2 alone. By these procedures we isolated mutants of all the classes mentioned above except pmi, and also mutants of several new classes, described below. Our data do not permit inference as to the relative frequencies of the different classes of mutants, because in many experiments we sought particular classes by selecting with mixtures of phages. Mutants representative of newly recognized classes and of previously described but unmapped classes were crossed with smooth Hfr donor strains, to determine the location of their $r f$. mutations. We describe below the various classes in the sequence of their known or inferred LPS defects, considering first those with the most superficial defects, i.e. the $r f c$ class, deficient in polymerization of $\mathrm{O}$ repeating units.

$r f c(O$-polymerase-defective) Mutants. In their cultural and serological properties these mutants resembled the $r f c$ mutants of Salmonella typhimurium and the SR recombinants from group $B \times$ group $C$ crosses, described above. The LPS of one of the newly-isolated mutants of pattern Zsr has been shown (Hämmerling, 1968) to contain small amounts of 


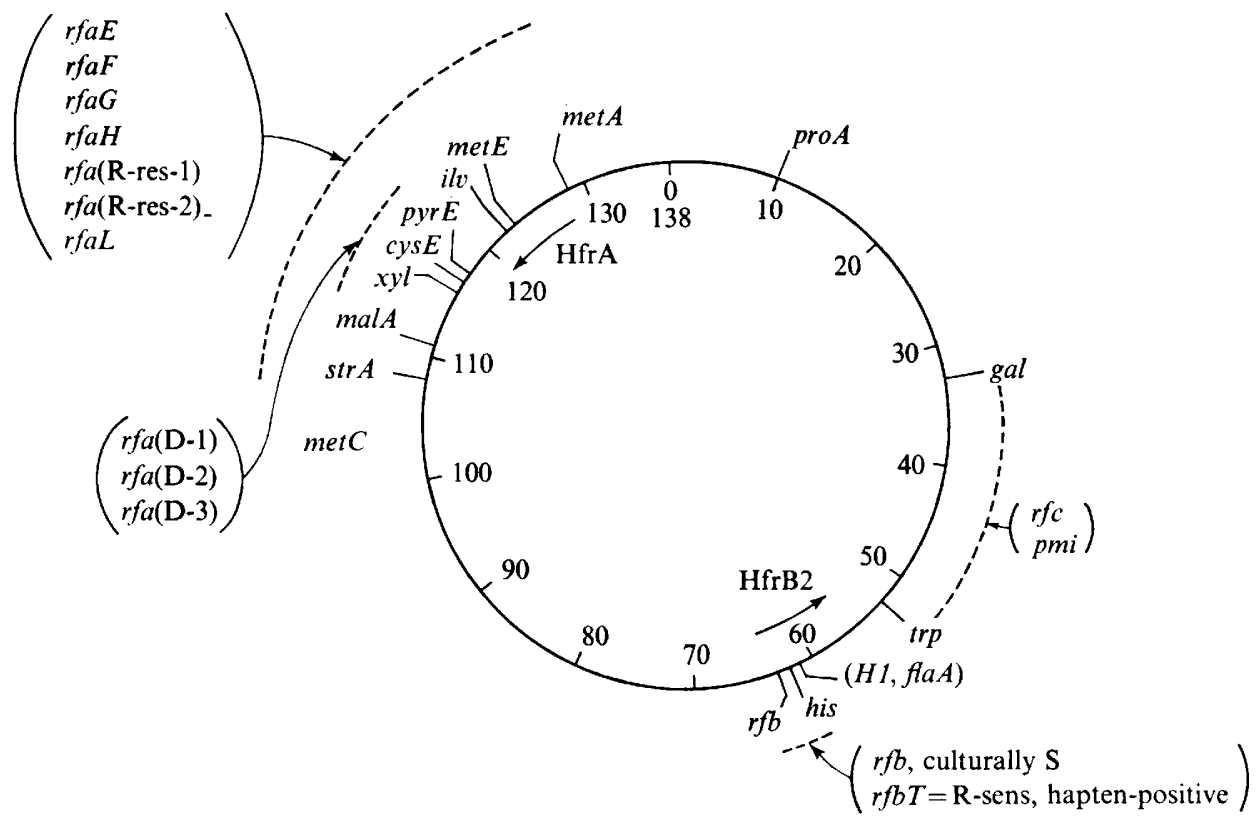

Fig. 2. Linkage map of Salmonella typhimurium. Map positions of standard loci, times in minutes and points of origin, etc. of Hfr strains from Sanderson (1970). Dotted lines are approximate map positions of loci concerned in biosynthesis of LPS, inferred from data in this paper, or by Wilkinson \& Stocker (I968) for $r f a G$ and $r f a H$, or by Subbaiah \& Stocker (1964) for $r f a$ loci now shown to include $r f a L, r f a(\mathrm{R}-\mathrm{res}-\mathrm{I})$ and $r f a(\mathrm{R}-\mathrm{res}-2)$. The relative order of loci shown within parentheses is unknown.

O-specific sugars, the ratio of heptose to rhamnose being about $1: 0 \cdot 6$, as in previously analysed SR strains (Naide et al. 1965). Analyses of the isotopically-labelled mannosecontaining oligosaccharides in partial acid hydrolysates of LPS from SLI IOI, an $r f c$ mutant deficient also in phosphomannoisomerase (see below), has confirmed the inference that the LPS side-chains of part-rough mutants of class $\mathrm{C}$, of resistance pattern $\mathrm{Zsr}$, indeed carry only single O-specific oligosaccharide units (Nikaido et al. 1966; Yuasa et al. 1970), as expected if they are $r f c$ mutants deficient in ability to polymerize $O$ repeating units. To map $r f c$, the biochemically-characterized $r f c$ mutant sL9or was crossed with a smooth strain, SL862 (= LT2 $\operatorname{trp} A$ hisD (ColEI) etc.) as donor, recombination being obtained by the use of colicin factors (Smith \& Stocker, 1962). The results of this cross, made in collaboration with $\mathrm{Dr}$ W. Shephard, indicated linkage of $r f c$ to $\operatorname{trp}$. The same $r f c$ recipient as next crossed with an S. typhimurium Hfr donor, SR 305, which is LT2 his galK metC HfrA which injects clockwise from II 9 min (Fig. 2). Selection was made for $t r p^{+}$of the donor and for $\operatorname{str} A$ of the recipient, a late marker for this $\mathrm{Hfr}$ donor. Of 53 recombinants $3 \mathrm{I}$ had no donor marker except $t r p^{+}$and so contributed no information. Of the remaining 22 recombinants six had the $r f c^{+}$of the donor, which suggests some linkage of $r f c$ to $\operatorname{trp}$. None of Io having the donor $\mathrm{fla}^{+}$and $h i s$-alleles had its $r f c^{+}$allele, which showed that $r f c$ is not within the segment $\operatorname{trp}$-flaA-his. None of the six trp ${ }^{+}$recombinants with the donor $r f \mathcal{c}^{+}$had its gal-allele, making the order $r f c-g a l-t r p$ less likely than $g a l-r f c-t r p$. One of the newly isolated $r f c$ mutants, SLI034, $r f c-465$, was similarly crossed with HfrA: the distribution of recombinant classes amongst $\operatorname{trp} p^{+}$str $A$ recombinants (Table 4 ) indicated that $r f c$ was between gal and $t r p$, this position requiring additional cross-overs in only two of the I Io recombinants analysed, whereas any other position for $r f c$ required additional cross-overs in at least nine recombi- 
Table 4. Mapping of $r f$ from segregation amongst $\mathrm{I}$ Io $\operatorname{trp} B^{+}$strA recombinants from cross of $\mathrm{Hfr} A$ donor to $\mathrm{rfc}$ recipient, SLIO34

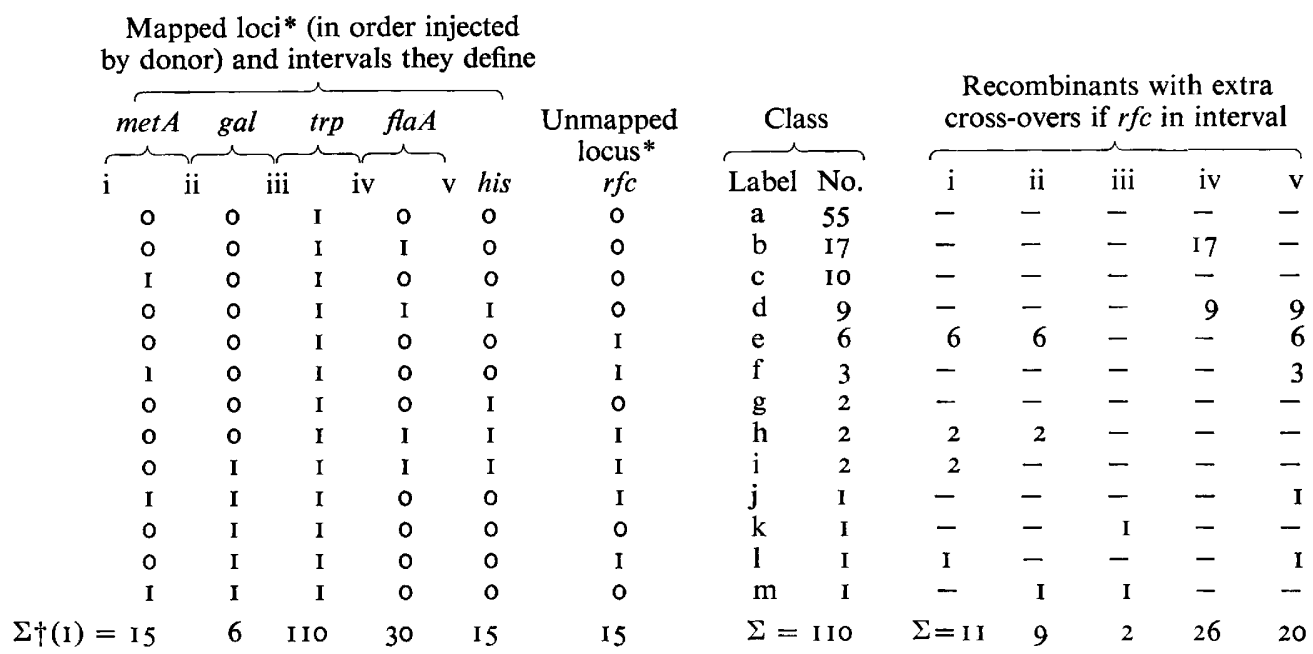

* Donor alleles represented by symbol I and recipient alleles by symbol O. Donor, SR305, was his galK metC HfrA. Recipient, sL1034, was metA trpB flaA strA rfc-465. The donor metC, which is leaky and temperature-sensitive, was not scored, but was probably unrepresented since it is transferred very late by HfrAsee Fig. 2.

$\dagger$ Total no. of recombinants with donor allele at indicated locus.

nants. Thus in two independently-isolated $r f c$ mutants of $S$. typhimurium LT2 the affected locus is between gal and trp. Mäkelä (1966) located the $r f c^{+}$locus of group B salmonellas in the same segment by analysis of recombinants from group B $(S$. abony $) \times$ group $\mathrm{CI}$ ( $S$. montevideo) crosses.

Phosphomannoisomerase-deficient mutants. No mannose-negative mutants were obtained by selection for resistance to phage $\mathrm{P}_{22}$.c2. We therefore sought such mutants by plating mutagen-treated cultures on MacConkey-type mannose indicator plates. Investigation of several non-fermenting colonies yielded two mannose-negative mutants which still fermented various other sugars. Assay of enzyme activity (Dr M. J. Osborn, personal communication) showed that both were defective in phosphomannoisomerase activity, SL 1030 (pmi-404) having $0.8 \%$ and SLI028 (pmi-402) $2.4 \%$ of wild-type activity (Table 5). The latter mutant was also leaky by cultural characters, for it grew slowly with mannose as the sole carbon source and produced an acid reaction in mannose peptone-water. Both mutants gave the Pmi phage pattern (with variable, partial sensitivity to P22.c2) on nutrient agar and a smooth pattern on mannose-supplemented nutrient agar. The mannose-negative mutant, SLI I I, pmi-408, was similarly obtained from an $r f c$ parent strain, SL9OI. It gave pattern Pmi on nutrient agar and pattern Zsr on mannose-supplemented agar.

To map pmi, mutant SL1030 was first crossed with donor SR305, which is LT2 gal his metC HfrA. Recombinants with the donor $\operatorname{trp}{ }^{+}$or $m e t A^{+}$and the recipient $s t r$ alleles were tested for pmi character by replication to mannose indicator plates and by tests of phage sensitivity, with results which always agreed. Velvet-pad replication of $\mathrm{I} 39 \mathrm{trp}^{+}$recombinants to mannose indicator plates showed that 26 of them had the donor $p m i^{+}$allele and $\mathrm{I} 6$ of these 26 (i.e. $62 \%$ ) also had the donor $\mathrm{gal}^{-}$allele, a result suggesting fairly close linkage of pmi and $\mathrm{gal}$. Analysis by least number of cross-overs indicated that pmi was probably between $g a l$ and 
Table 5. Properties of phosphomannoisomerase-deficient mutants

\begin{tabular}{|c|c|c|c|c|c|c|c|c|}
\hline \multirow[b]{2}{*}{$\begin{array}{c}\text { Strain } \\
\text { no. }\end{array}$} & \multirow{2}{*}{\multicolumn{2}{|c|}{ LPS genotype }} & \multicolumn{4}{|c|}{ Mannose metabolism } & \multicolumn{2}{|c|}{ Phage pattern } \\
\hline & & & $\begin{array}{l}\text { Isomerase } \\
\text { activity* }\end{array}$ & $\begin{array}{c}\text { Mannose } \dagger \\
\text { peptone } \\
\text { water }\end{array}$ & $\begin{array}{c}\text { MacConkey } \dagger \\
\text { mannose } \\
\text { agar }\end{array}$ & Utilization $\ddagger$ & $\begin{array}{l}\text { On nutrient } \\
\text { agar }\end{array}$ & $\begin{array}{c}\text { On nutrient } \\
\text { agar }+0.5 \% \\
\text { mannose }\end{array}$ \\
\hline LT2 & $r f^{+}$ & $p m i^{+}$ & 25 & A & A & + & Smooth & Smooth \\
\hline SLIO28 & $r f .^{+}$ & pmi-402 & 0.6 & A & - & + & Pmi & Smooth \\
\hline SLIO3O & $r f^{+}$ & pmi-404 & 0.2 & - & - & - & Pmi & Smooth \\
\hline SLIIOI & $r f c-497$ & pmi-408 & $\S$ & - & - & - & Pmi & $\mathrm{Zsr}$ \\
\hline
\end{tabular}

* Phosphomannoisomerase activity in units/mg protein (Rosen et al. 1965). Data of Dr M. J. Osborn (personal communication).

$\dagger$ Reaction in peptone water with I \% mannose or on mannose MacConkey agar after 2 days at $37^{\circ}$. $A=$ acid, $-=$ no acid. (All strains fermented glucose, maltose, mannitol, fructose and sorbitol.)

$¥$ Growth after two days at $37^{\circ}$ on solid defined medium with $0.2 \%$ mannose as carbon and energy source.

$\S$ Almost no activity (H. Nikaido, personal communication).

trp or between $\operatorname{trp}$ and $f a A$, since most of the recombinants with the donor $t r p^{+}$and $p m i^{+}$ alleles retained the recipient $\mathrm{flaA}^{-}$and his $^{-}$alleles. Further information was obtained from a cross of SLI Ior, the $r f c$ pmi double mutant, with the same HfrA donor. In the analysis it was assumed that $r f c$ was between $g a l$ and trp. Amongst 27 recombinants selected as $t r p^{+}$the representation of donor alleles was five for $p m i$, four for $g a l$ and four for $r f c$, and suggested that pmi is linked to $g a l$ and $r f c$; but the data gave no indication as to the order of these three loci. Velvet-pad replication of about $775 \mathrm{met}^{+}$recombinants from the same cross showed that 32 were $p m i^{+}: 20$ of these 32 had the donor $r f c^{+}$and 12 the donor $g a l^{-}$alleles, which indicates linkage of pmi to gal and $r f c$. Cross-over analysis did not indicate the relative order of these three loci. If the pmi loci involved in the two crosses are the same then it seems that $p m i$ is between $g a l$ and $t r p$, probably nearer to $g a l$ than to $t r p$; but its position relative to $r f c$, which also falls in the gal-trp sector, is not apparent.

Hapten-positive rfb mutants. Eleven of 20 mutants of pattern R-sens, isolated from SLI092, had the usual cultural and serological characters of rough mutants. A screening test using an Salmonella typhimurium $\mathrm{HfrB} 2$ donor showed that in nine of Io such mutants tested the site of the $r f$. mutation was close to his; we inferred that these were $r f b$ mutants. (The exceptional strain, due to mutation not linked to his, was not further investigated; it may have been an $r f a$ mutant of the type which are of $R$-sens phage pattern.) Extracts of these nine mutants were tested for the presence of O-specific 'hapten' (see Methods): three mutants gave strongly positive ring tests, whereas the others were negative. Thus the three mutants SLII96, SLII97 and SLII98, unlike other $r f b$ mutants but like $r f a$ mutants, accumulate $\mathrm{O}$-specific polysaccharide, and we therefore term them hapten-positive $r f b$ mutants. Tests on one of these mutants, SLII96, in the laboratory of Dr M. J. Osborn have shown that its LPS, like that of typical $r f b$ mutants, accepts O-specific polymer when incubated in an in vitro system with broken-cell preparations of an $r \mathrm{fb}^{+}$strain; this shows that SLI 196 makes a 'complete core' LPS. Correspondingly, broken-cell preparations of SLI I96 are defective in enzymic activity for transferring $\mathrm{O}$ chains from antigen carrier lipid to complete core (Cynkin \& Osborn, I968 and personal communication). We infer that the locus affected in hapten-positive $r f b$ mutants is concerned with this O-transferring reaction, and we term it $r f b T$.

Culturally smooth rfb mutants. Nine of the 20 mutants of pattern R-sens obtained from SLI092 had cultural and serological properties approximating to smooth, despite their 
Table 6. Sugar composition and complement-fixing ability of LPS and LI fractions from non-smooth mutants. (Data of K. Nakane and H. Nikaido, personal communication)

LPS fraction*

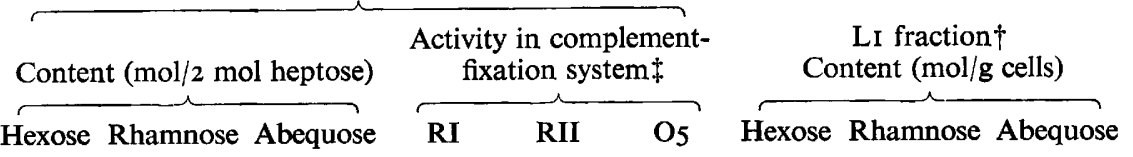

Strain no. Hexose Rhamnose Abequose $\overbrace{\text { RI }}$ RII O5 $_{\text {Hexose Rhamnose Abequose }}$

$r f b$, culturally smooth mutants

$\begin{array}{lrrrrrrrrr}\text { SLIII2 } & 4.1 & 0.06 & 0.19 & - & +++ & \text { n.t. } & 14.9 & <0.2 & <0.3 \\ \text { SLIII3 } & 3.3 & <0.02 & <0.02 & + & +++ & \text { n.t. } & 18.3 & <0.2 & <0.3 \\ \text { SLIII4 } & 3.4 & <0.02 & <0.02 & + & \text { n.t. } & \text { n.t. } & 13.3 & <0.2 & <0.3 \\ \text { rfb (control) } & & & & & & & & & \\ \text { TVI57 } & 2.7 & 0.03 & <0.02 & - & +++ & \text { n.t. } & \text { n.t. } & \text { n.t. } & \text { n.t. }\end{array}$

Class D, pattern D-I mutants

$\begin{array}{llllllllll}\text { SL733 } & 2.8 & 0.3 & 0.2 & - & + & +++\S & \text { n.t. } & \text { n.t. } & \text { n.t. } \\ \text { SLI035 } & 4.2 & 0.27 & 0.28 & + & + & \text { n.t. } & & & \\ \text { SLI036 } & \text { n.t. } & \text { n.t. } & \text { n.t. } & \text { n.t. } & \text { n.t. } & +++\S & \text { n.t. } & \text { n.t. } & \text { n.t. }\end{array}$

Class D, pattern D-2 mutants

$\begin{array}{llllllllll}\text { SLIIII } & 3.9 & 0.23 & 0.30 & +++ & - & \text { n.t. } & \text { I7.I } & 3.2 & 2.7 \\ \text { SLII5O } & 4.8 & \text { I.13 } & \text { I.37 } & + & \text { n.t. } & \text { n.t. } & \text { I4.3 } & \text { I.7 } & 1.8 \\ \text { SLII5I } & 2.3 & 0.27 & 0.24 & +++ & - & \text { n.t. } & \mathbf{2 5 . 2} & 3.8 & 3.6\end{array}$

$r f a$, pattern R-sens (control)

\begin{tabular}{|c|c|c|c|c|c|c|c|c|c|}
\hline TV226 & $3 \cdot 7$ & $<0.02$ & $<0.02$ & - & +++ & n.t. & I I 4 & $\mathbf{I} \cdot \mathbf{2}$ & $I \cdot 0$ \\
\hline \multicolumn{10}{|c|}{$f a$, pattern R-res-I (control) } \\
\hline TV16o & $2 \cdot 8$ & 0.20 & 0.18 & +++ & - & n.t. & $13 \cdot 6$ & $\mathrm{I} \cdot 8$ & $2 \cdot 3$ \\
\hline TVI6I & $3 \cdot 4$ & $0 \cdot 14$ & 0.19 & +++ & - & n.t. & $14 \cdot 1$ & $2 \cdot I$ & $1 \cdot 9$ \\
\hline \multicolumn{10}{|c|}{$c$ (control) } \\
\hline SL9OI & $3 \cdot 2$ & 0.5 & 0.4 & - & - & $+++\S$ & n.t. & n.t. & n.t. \\
\hline SL954 & $2 \cdot 7$ & 0.3 & 0.2 & n.t. & n.t. & $+++\S$ & $12 \cdot 0$ & $<0.2$ & $<0.3$ \\
\hline
\end{tabular}

* LPS extracted by hot-phenol-water method and purified by repeated ultracentrifugation.

$\dagger$ LI fraction is the supernatant of the first ultracentrifugation of dialysed hot-phenol-water extract and contains the O-specific 'hapten' if present in the hot-phenol extract (Beckmann et al. I964). The high hexose content of all Li fractions tested probably represents a glucan.

$\ddagger$ For the quantitative complement-fixation test used see Naide et al. (I965). RI and RII are rough antigenic specificities, RII being characteristic of the 'complete core' LPS of $r f b$ mutants (Beckmann et al. 1964).,+++++ and $+=$ extent of complement fixation; $-=$ absence.

$\S$ The amount of LPS needed for maximum complement fixation is about eight times greater than the amount of smooth LPS needed for maximum fixation.

n.t. $=$ not tested.

characteristic rough phage pattern. Their colonies on nutrient agar were smooth-looking and of buttery consistency, they gave uniform turbidity when grown in broth and did not agglutinate in saline $(\mathrm{NaCl} 0.9 \%)$ in slide tests but were strongly agglutinated by anti-4, I 2 serum. Tests by Dr P. H. Mäkelä showed that representative mutants were strongly agglutinated by anti-4, I 2 sera completely absorbed with an $r f b$ strain and that three mutants, SL I I 2 , SLIII 3 and SLIII4 removed 4, I2 antibody from an O-antiserum nearly as efficiently as did smooth cells. Biochemical investigations of several mutants (K. Nakane and H. Nikaido, personal communication and Table 6; Hämmerling, 1968) showed that their LPS resembled the 'complete core' LPS of typical $r f b$ mutants, both in their content of core sugars and by their serological specificity, RII (Beckmann et al. 1964), but differed in that they showed also smooth serological specificity and (in some mutants at least) contained detectable amounts of 
O-specific sugars. The 'non-sedimentable' Li fractions (Beckmann et al. I964) of the phenol extracts of the mutants contained no significant amount of $\mathrm{O}$-specific sugars (Table 6), in contrast to those from $r f a$ control strains; thus these 'culturally smooth' R-sens mutants resemble $r f b$ mutants by failure to accumulate O-specific hapten.

For genetic analysis, six mutants were crossed with an HfrB2 smooth donor, su396. In each cross, 82 to $92 \%$ of recombinants with the donor $h^{+}{ }^{+}$were of smooth phage pattern, which indicates close linkage of the phage resistance character to his.

The chemical and serological character of the LPS of several culturally smooth $r f b$ mutants and their failure to accumulate hapten suggest that they make 'complete core' LSP but have almost complete defects in biosynthesis of O-specific side-chains for attachment to it. Presumably they have somewhat leaky mutations affecting genes of the his-linked $r f b$ gene cluster.

Mutants of patterns $R$-res-I and R-res-2. Many of the newly isolated mutants gave the phage resistance pattern R-res-I (I 7 mutants) or R-res-2 (five mutants), already encountered amongst the $r f a(=\operatorname{rouA})$ mutants of Subbaiah \& Stocker (1964), and were therefore considered to be $r f a$ mutants. Except for four mutants found to be sensitive to bile-salts (as described below) they were not further investigated.

Part-rough mutants of patterns $D-1, D-2$ and $D-3$. Many mutants proved to be sensitive to several rough-specific phages, resistant to Felix $O$ phage but sensitive, at least in part, to one or more smooth-specific phages, thus resembling the class D part-rough mutant SL733 (= st/22, Yamamoto \& Anderson, 196I), described above. Several mutants gave the same phage pattern, D-I, as SL733. Others differed only by their resistance to phage 6SR (and SI3 and $\phi \mathrm{X}-\mathrm{I} 74$ which, in our experiments, had the same host range), a pattern we termed D-2. One mutant had a phage pattern identical with D-2 except that it was resistant to phage $\mathrm{Br}$, a combination we call pattern D-3 (Table 3). The newly-isolated mutants of patterns D-I, D-2 and D-3, like the original part-rough class D mutant SL733, gave smooth-looking colonies and turbid broth cultures with little deposit and were agglutinated by anti-O sera in slide tests.

To map the mutations determining phage-patterns D-1, D-2 and D-3, we mated strain SLI035, a class D-I mutant of SL696 (= LT2 met $A \operatorname{trp} B$ flaA str $A$ etc.) with the smooth HfrB2 donor, su396 (Fig. I). Since most recombinants with the donor $m e t A^{+}$had also the $\operatorname{str} A^{+}$allele and the smooth phage pattern of the donor, we concluded that $r f .-469$, determining phage pattern D-I of SL1035, was in or close to the strA-metA region.

More precise mapping necessitated the use of class D mutants with additional markers in the upper left quadrant of the map. Several class D mutants were isolated from: SLI027, a xylose-negative $(x y l)$ and $m e t E$ (see footnote, Table I) mutant of sL696; SLI093, a malA mutant of SLI027; and SLI094, an ilv mutant of SLI027 (Table I). The $r f$. locus of strain SLI036, a class D-I mutant derived from SL 1027, proved to be closely linked to $x y l$ : in one cross, 34 of $4 \mathrm{I} x y l^{+}$recombinants also had the smooth phage pattern of the donor, whereas only 28 of the $4 \mathrm{I}$ had the donor $\operatorname{str} A$ allele and only I 7 its $m e t A^{+}$(and $m e t E^{+}$) alleles. Crosses of several other class D-I mutants with $\mathrm{HfrB} 2$ likewise indicated close linkage of the $r f$. loci to $x y l$ (Table 2); in two crosses in which malA was segregating the $r f$. sites concerned mapped between $\mathrm{malA}$ and $\mathrm{met} A$.

Crossing of a class D-I HfrB2 donor with a smooth $x y l$ recipient also showed close linkage of its $r f$. locus to $x y l$. If all these class D-I mutants result from mutation at a single locus it must lie between malA (at I I $2 \mathrm{~min}$ ) and the origin of HfrA (at I I9 min), probably between $x y l$ (at II6 min) and II9 min (Fig. I). As some $r f a$ loci have been shown to be co-transducible with cysE and with pyrE (Kuo \& Stocker, I968; Sanderson \& Saeed, I968) 
a smooth pyrE xyl rha line, SL2229, was prepared and a class D-r mutant isolated from it; this mutant was crossed with HfrB2, selection being made for the donor $x y l^{+}$and $p y r E^{+}$ alleles, or for its $p y r^{+}$and $r h a^{+}$alleles. Each selection yielded a high proportion of smooth recombinants; the order requiring the least number of cross-overs is $x y l-r f$. $-p y r E$.

Two mutants of class D-2 and one of class D-3 were analysed genetically by crossing with smooth $\mathrm{HfrB} 2$, selection being made for the donor $x y l^{+}$or $m e t A^{+}$(and met $E^{+}$alleles). As in the case of D-I mutants there was close linkage of $r f$. to $x y l$ but the data did not permit distinction between the orders $\operatorname{str} A-r f .-x y l-m e t A$ and $\operatorname{str} A-x y l-r f .-m e t A$.

Thus in all of at least I I part-rough class $D$ mutants, including strains giving patterns D-I, D-2, and D-3, the $r f$. sites affected are in the $\operatorname{str} A-$ mal $A-x y l-m e t A$ region, previously shown to contain $r f a$ loci (Subbaiah \& Stocker, 1964) determining the resistance patterns R-res-I and R-res-2, and in several more precisely mapped class D mutants the data are compatible with mutation within the short cysE-pyrE region, shown by the test of cotransduction to contain several $r f a$ loci. The LPS of six part-rough class D mutants contained small amounts of O-specific sugars (K. Nakane \& H. Nikaido, personal communication and Table 6; Hämmerling, I968). LPS from two class D-I mutants did not give strong complement fixation in either an anti-RI or an anti-RII system: those from two of the three class D-2 mutants tested reacted strongly in the RI system (Table 6). The Li ('non-sedimentable') fraction of phenol extracts of all three class D-2 mutants contained O-specific sugars, showing that these three mutants accumulate hapten (the class D-I mutants were not tested in this respect). Thus evidence from analyses of phenol extracts, both LPS and non-sedimentable fractions, support the inference that class D-I and D-2 mutants have lesions which reduce the amount of O-specific material added to the LPS core, and are compatible with the suggestion, from genetic analysis, that the mutations concerned are at $r f a$ loci, involved in biosynthesis of LPS core.

Mutants sensitive to phage $C_{2} \mathrm{r}$, of classes $r f a G, r f a H$ and galE. As described by Wilkinson \& Stocker (I968) some phage-resistant mutants were sensitive to phage C2 I (which was known to attack Salmonella typhimurium mutants blocked in synthesis of UDPgalactose), yet they retained ability to ferment galactose, which showed they could still make UDPgalactose. Two of ro such mutants isolated from either smooth strains, or pmi or $r f b$ mutants were of phage pattern Epi-I, characteristic of mutants lacking UDPgalactose-epimerase (Table 3). The other eight showed a new pattern, Epi-2, differing from Epi-I only by resistance to phage $\mathrm{Br} 2$ (Table 3). Biochemical investigation (Osborn, 1968) showed that extracts of the two mutants of pattern Epi-I lacked the ability to attach the main-chain galactose unit of the core side-chain to galactose-deficient LPS and that extracts of several mutants of pattern Epi-2 were, instead, unable to catalyse the attachment of the proximal glucose unit to glucose-deficient LPS. The loci affected may be the structural genes for corresponding glycosyl LPS transferases (see Discussion for other possibilities in the case of $r f a H)$. Both loci, $r f a H$ concerned with formation of the main-chain galactose unit and $r f a G$ with that of the proximal glucose unit, were found to map in the vicinity of $x y l$ (Wilkinson \& Stocker, 1968).

The same selections gave four galactose non-fermenting, C2I-sensitive mutants, three from smooth parent lines and one from an $r f b$ strain. On nutrient agar all four mutants gave the pattern Epi-I, characteristic of mutants deficient in UDPgalactose-4-epimerase (see above). Growth on galactose-supplemented medium restored the parental, smooth, phage sensitivity pattern of two of the mutants, which presumably retain (at least in part) the ability to take up galactose from the medium and convert it via galactose-phosphate to UDPgalactose. Only one of these two mutants showed the galactose-sensitivity typical of 
mutants deficient only in epimerase. The phage pattern of the other two galactose-negative mutants was unaltered by the presence of galactose in the medium; they thus resemble mutant gal-5 (Fukasawa \& Nikaido, 1961), deficient in all three enzymes of the galactose pathway. We did not investigate these mutants genetically.

Bile-salt sensitive mutants. Rough mutants with LPS lacking heptose (and all sugars distal to it) have been described in Salmonella minnesota and S. ruiru. Four such mutants, strains mR595, mR613, mRI000 and rRI28 (Lüderitz et al. I966), were obtained from Dr O. Lüderitz. All of them except rRI 28 failed to grow on media containing bile salts, for instance MacConkey medium. Examination of our rough mutants of $S$. typhimurium showed that four of them, SLIIO2, SLII8I, SLII82 and SLII83, were sensitive to bile salts, since they failed to grow, or grew very poorly, on MacConkey medium or on nutrient agar or defined medium containing sodium deoxycholate, $0.1 \%$ or more. Three of them, SLI I8I, SLII 82 and SLII 83 had the phage pattern R-res-2; the other, SLIIO2, differed only in that it gave variable reactions with phage Ffm (usually plaques, instead of confluent lysis) and P22 I.c2 (confluent lysis or plaques or no action)-we term the combination of bile-salt sensitivity with these phage sensitivity patterns Hep-I and Hep-2 (Table 3). Strain SLIIO2 grew abnormally on nutrient agar, the colonies after $24 \mathrm{~h}$ at $37^{\circ}$ being heterogeneous in size, mostly very small and glassy-looking. Phase-contrast microscopy of bacteria from nutrient agar plates incubated for one or two days showed many cells of abnormal, coccobacillary, morphology. The other three bile-salt-sensitive $S$. typhimurium mutants and the heptosenegative mutants in other species received from Dr Lüderitz did not show this aberration.

Acetone-dried cells of SLI IO2 were sent to Dr O. Lüderitz and Dr G. Hämmerling (MaxPlanck Institute for Immunobiology, Freiburg, Germany) and phenol extracts were made in the same laboratory from the residues after alcohol extraction of large crops of strains SLI I02, SLI I 8I and SLI I 82, grown as sources of sugar nucleotides (Ginsburg, I966; Scher \& Ginsburg, 1968). The main results of these investigations are summarized in Table 7 (Hämmerling, I968; E. Ruschmann \& O. Lüderitz, personal communication). The LPS of strain SLI IO2 contained no detectable heptose and no more than traces of glucose, galactose and O-specific sugars; haemagglutination-inhibition tests showed it to be serologically similar to the Re (heptose-deficient) LPS of Salmonella minnesota mutants mR595 and mR6I3. Hydrolysates of the LI fraction of the same phenol extract contained glucose, galactose and the three O-specific sugars. LPS from mutants SLII8I and SLII82 likewise lacked glucose, galactose and $\mathrm{O}$-specific sugars but contained some heptose and were serologically similar to the Rd 2-type LPS of $S$. minnesota and $S$. ruiru mutants deficient in formation of the distal of the two heptose units of the core side-chain (Risse et al. 1967). The fourth of our bile-salt-sensitive mutants, SLI I83, appears to be a leaky rough mutant, since its LPS contained some O-specific sugars (rhamnose : heptose ratio about $0 \cdot 7: 1$ ) and had some smooth serological activity. The Rd2 serological activity and KDO:heptose ratio of its LPS indicate that the incomplete block in strain SLII 83 is in formation of the distal heptose unit.

Mutant SLIIO2 was crossed to an HfrB2 donor strain and recombinants were scored for smooth or rough character, both by their phage sensitivities and by testing their ability to grow in the presence of bile-salts, with results which in all cases agreed. The $r f$. locus of SLI IO2 appeared linked to $x y l$ and to $m e t A, 86 \%$ of recombinants selected for possession of the donor $x y l^{+}$and $82 \%$ of those selected for possession of donor met $^{+}$(and $m e t E^{+}$) being smooth. In each selection many recombinants with the donor $r f .^{+}$allele derived the whole str $A-x y l-m e t E-m e t A$ region from the donor, and in consequence analysis by least number of cross-overs gave no definite indication of the location of the $r f$. site within the region tre 
Table 7. Properties of LPS from bile-salt-sensitive rough mutants of Salmonella typhimurium LT2. (Data of Hämmerling (1968) and of E. Ruschmann \& O. Lüderitz, personal communication)

\begin{tabular}{|c|c|c|c|c|c|c|c|c|c|}
\hline \multirow{2}{*}{$\begin{array}{c}\text { Strain and } \\
\text { mutation no. }\end{array}$} & \multicolumn{5}{|c|}{ Content, $* \%$ by wt } & & \multicolumn{3}{|c|}{ activity in system } \\
\hline & KDO & Heptose & Glucose & Galactose & Rhamnose* & $P$ & RdI & $\mathbf{R d} 2$ & $\operatorname{Re}$ \\
\hline SLIIO2, rfa-543 & 19 & trace & 0.5 & 0.2 & - & $2 \cdot 5$ & . & & 8,15 \\
\hline SLII $8 \mathrm{I}, r f a-5 I I$ & 17.9 & $7 \cdot 3$ & 0.2 & 0.2 & - & $2 \cdot 4$ & . & $8-3 I$ & \\
\hline SLII $82, r f a-537$ & 16.5 & $6 \cdot 0$ & 0.5 & 0.5 & - & $I \cdot 4$ & . & I6-3I & \\
\hline SLI I $83, r f a-546$ & IO & $5 \cdot 2$ & 0.8 & $2 \cdot 7$ & $3 \cdot 4$ & $1 \cdot 7$ & . & $2-16$ & \\
\hline
\end{tabular}

* The LPS samples were obtained from hot-phenol-water extracts of batches of cells (some of which had previously been extracted with ethanol, see text).

$\dagger$ Lowest concentration(s), in $\mu \mathrm{g} / \mathrm{ml}$, of LPS inhibiting agglutination of red cells coated with LPS of indicated type by homologous rabbit antibacterial sera; for the $\mathrm{Rd} 2$ systems tested the figures are the range of minimal inhibitory concentrations.

.$=$ No inhibition at $250 \mu \mathrm{g} / \mathrm{ml}$; n.t. $=$ not tested. Sources of LPS: mR7 for system RdI ; mR3, mR4, rR3 and/or mR597 for system Rd2; and mR595 and mR6r3 for system Re.

$\ddagger-=$ Not detected. Qualitative tests for mannose and abequose gave similar results, i.e. positive for strain SLI I 83 and negative for the other three strains.

( $58 \mathrm{~min}$ ) to $\mathrm{met} A$ (I $29 \mathrm{~min}$ ), though somewhat favouring the region between $x y l$ (I I $6 \mathrm{~min}$ ) and met $A$ ( $29 \mathrm{~min}$ ). Crosses of an $\mathrm{HfrB} 2$ donor to the other three bile-salt sensitive mutants gave essentially similar results.

Other classes of phage-resistant mutants. Three mutants selected from smooth parent strains by exposure to phage Felix $O$ or phage Felix $O+$ phage Ffm were found to be resistant to Felix $O$ phage and to all the rough-specific phages but still sensitive to the three smooth-specific phages - a pattern we call For (Felix O-resistant). These three mutants were smooth in cultural properties and agglutinable by anti-O sera and were not investigated further, since they did not seem to be non-smooth mutants. Mutants that produced large mucoid-looking colonies on several media (nutrient agar, defined medium, MacConkeytype agar) after overnight incubation at $37^{\circ}$ were encountered among phage-resistant mutants isolated from smooth and non-smooth parent strains. Non-mucoid 'revertants' accumulated rapidly during storage of some of the mucoid mutants. The phage-resistance pattern of mucoid mutants was identical with that of their non-mucoid parents if the nutrient agar test plates were examined after 4 to $6 \mathrm{~h}$ of incubation at $37^{\circ}$; after longer incubation the areas where phage had been applied were obscured by mucoid growth. We did not further investigate these mutants.

\section{DISCUSSION}

Tests on their sensitivity to a number of phages, and to the growth-inhibiting effect of bile-salts, enabled us to recognize sensitivity patterns characteristic for various previously reported classes of non-smooth mutants of Salmonella typhimurium and to distinguish several new classes amongst mutants selected for resistance to one or more phages.

Previously encountered mutants and inter-group hybrids intermediate between smooth and rough of the phenotype indicated semirough (SR) or class $\mathrm{C}$, and inferred unable to polymerize $O$ repeating units, were found to have a distinctive phage pattern, Zsr (formerly $\mathrm{Zpr}$, being resistant to smooth-specific phages, sensitive to Felix $\mathrm{O}$ phage and resistant to all rough-specific phages except P22I (Table 3). Four mutants of this pattern were encountered amongst those selected for resistance to phage P22. The new mutants resembled 
previously investigated SR mutants and recombinants in their serological and cultural properties (and, in the one mutant tested, in respect of presence of O-specific sugars in their LPS) and were therefore considered to be $r f c$ mutants, deficient in polymerization of $\mathrm{O}$ repeating units. The site of mutation in two $r f c$ mutants, determined by Hfr crosses, was between $g a l$ and trp; Mäkelä (1966) inferred, from analysis of inter-group crosses, that the $r f c^{+}$locus of group B and group D salmonellas mapped in this section. The O-polymerizing enzyme must differ between certain $O$ groups, in particular between groups having galactose as reducing terminus and groups with some other sugar at the reducing end of the $O$ repeat unit. The location of the $r f c$ locus (presumed to be the structural gene for the O-polymerase in group B and group D), in the gal-trp segment, remote from the $r f b$ cluster, thus constitutes an exception to the generalization that all the 'special' enzymes required only for assembly of the $\mathrm{O}$ side-chains of a given $\mathrm{O}$ group map in a single cluster, at $r f b$.

Mutants lacking phosphomannoisomerase, called pmi, are unable, unless supplied with exogenous mannose, to synthesize GDPmannose, the precursor of the mannose of the $\mathrm{O}$ repeating unit of group B salmonellas (Rosen et al. 1965). Four such mutants had a distinctive sensitivity pattern, Pmi, being sensitive to Felix $O$ phage and all the rough-specific phages except $\mathrm{C}_{21}$ and almost completely resistant to smooth-specific phages. This corresponds to the sensitivity pattern of $r f b$ mutants (unable to synthesize $\mathrm{O}$ repeat units and therefore making a complete core LPS lacking $O$ side-chains) if one attributes the slight degree of sensitivity to smooth-specific phages to leakiness of the phosphomannoisomerase defect. Growth in the presence of mannose restored sensitivity to smooth-specific phages (Rosen et al. 1965), and resulted also in complete or almost complete loss of sensitivity to rough-specific phages. Three pmi mutants, encountered amongst several mannose-nonfermenting mutants, resembled those previously examined both in their phage-sensitivity patterns and in the effect of supplementing the medium with mannose, which restored the parental sensitivity pattern. Hfr crosses indicated that the site of mutation in two newly isolated pmi mutants was near gal and trp, probably between these two loci: its relation to $r f c$, also located in this segment, could not be determined from the data. In Escherichia coli a locus determining phosphomannoisomerase, termed man, maps between trp and his (Markovitz, Sydiskis \& Lieberman, I967; Taylor, 1970). If these two genes correspond, i.e. if each is the structural gene for the enzyme, the situation constitutes an exception to the usual similarity of the linkage maps of two genera. The $\operatorname{trp}-c y s B-p y r F$ segment is known to be inverted between the two genera (Sanderson \& Hall, 1970) and the inverted region comprises also galU (T. Kuo, D. G. MacPhee, V. Krishnapillai \& B. A. D. Stocker, unpublished observations). If $p m i$ falls within the inverted region the difference in location in the two genera would be explained.

All the $r f b$ mutants of Subbaiah \& Stocker (1964), mutated near his and inferred to have defects in biosynthesis of O-specific polymer (Beckmann et al. 1964; Nikaido et al. 1964) showed the same pattern of phage sensitivity, termed R-sens, being resistant to smoothspecific phages and sensitive to Felix $O$ phage and to all our rough-specific phages except C2I. Several classes were distinguishable amongst 20 mutants of pattern R-sens isolated from a mutagen-treated culture of a smooth parent strain. Six mutants with the usual cultural properties of rough strains resulted from mutation near his and did not yield O-reactive material on extraction with trichloracetic acid: they were inferred to be $r f b$ mutants. Three mutants, with the cultural properties of rough mutants, arose by mutation near his but contained O-specific material extractable by trichloracetic acid. Biochemical examination (Cynkin \& Osborn, 1968; M. J. Osborn, personal communication) has shown that the LPS of these three mutants accepts $\mathrm{O}$-specific chains in a test in vitro, in this respect resembling 
the complete core LPS characteristic of $r f b$ mutants; and that, in the case of the only one of the three mutants so far tested, broken-cell preparations are at least partly deficient in ability to catalyse transfer of $O$ chains from antigen carrier lipid to complete core LPS. It thus seems that these mutants result from mutation at a locus (or loci) concerned with enzymic transfer of O chains from antigen carrier lipid to LPS core; the abbreviation $r f b T$ has been proposed (Mäkelä \& Stocker, I969) for the his-linked locus concerned. Six of I00 $h_{i s^{+}}$transductants evoked from SLII97 ( $f$ fbT604) were found to be smooth, as judged by phage sensitivity (Gemski \& Stocker, 1967): this frequency of co-transduction of $r f b T$ with his $D$ suggests that the $r f b T$ locus may be part of the main $r f b$ gene cluster.

Nine mutants of pattern R-sens resembled smooth strains in their cultural properties and agglutinability by anti-O sera: in six of them which were analysed genetically the site of mutation was close to his. The O-agglutinability and capacity to adsorb O-antibody, (P. H. Mäkelä, personal communication), of these mutants indicates that their LPS contains O-specific material, and O-specific sugars have been detected in LPS from some of them. We assume them to be leaky $r f b$ mutants, comparable to the leaky $r f a$ mutants discussed below.

Differences in sensitivity to phages defined three classes amongst the rfa mutants of Subbaiah \& Stocker (1964), which result from mutation in the str-metA region and are inferred to have defects in biosynthesis of the LPS core. Four mutants, previously noted to be sensitive to Felix $O$ phage and phage $6 \mathrm{SR}$, were of pattern R-sens - thus resembling $r f b$ mutants, known to manufacture complete core LPS. The LPS of these four $r f a$ mutants has since been shown to contain the $N$-acetylglucosamine unit (O. Lüderitz, personal communication) and to accept $\mathrm{O}$ chains from antigen carrier lipid in a test in vitro (M. J. Osborn, personal communication). Thus $r f a$ mutants of pattern R-sens, like $r f b T$ mutants (see above), are deficient in enzymic transfer of $O$ chains from their site of synthesis to the LPS core. The symbol $r f a L$ has been proposed for this locus, recently shown to map between $c y s E$ and pyrE (Kuo \& Stocker, I968).

Sensitivity or resistance to phage Br2 divided the eight $r f a$ mutants noted by Subbaiah \& Stocker (1964) to be resistant to Felix $O$ and 6SR phages into two classes: seven mutants were resistant to $\mathrm{Br} 2$ (pattern R-sens-I) and one mutant was resistant to $\mathrm{Br} 2$ (pattern R-res-2). Many mutants of pattern R-res-I and R-res-2 were encountered in this investigation but were not further investigated, and mutants of both these patterns have been encountered by Lindberg \& Holme (1968). The LPS of both R-res-I and R-res-2 mutants lack the acetylglucosamine unit, but contain some glucose and galactose (Beckmann et al. 1964; $\mathrm{O}$, Lüderitz, personal communication); the two sorts of LPS differ serologically (O. Lüderitz, personal communication; Lindberg \& Holme, I968). It has been surmised (Mäkelä \& Stocker, I969) that R-res-I mutants are deficient in a transferase needed for addition of the acetylglucosamine unit (class $r f a K$ ) and that R-res-2 mutants are deficient in the transferase for addition of the second glucose unit of the core side-chain (class $r f a J$ ). However recent structural studies (Hellerqvist \& Lindberg, I97I) on the LPS of strain TVI6o, an rfa mutant of pattern R-res-I (Subbaiah \& Stocker, 1964), show that its core side-chains terminate with the $\alpha \mathrm{I} \cdot 3$-linked, main-chain galactose unit (gal I), which implies that it is deficient in addition of the distal glucose unit. If so on the scheme of nomenclature proposed by Mäkelä \& Stocker (I969) the locus affected in mutants of pattern R-res-I should be termed rfaJ; but for the moment it may be non-comittally designated $r f a$ (R-res-I). In the same investigation it was shown that strain TVI 48, an $r f a$ mutant of pattern R-res-2, makes LPS core side-chains which lack the $\alpha \mathrm{I} \cdot 3$-linked galactose unit (some of them lacking also the $\alpha \mathrm{I} \cdot 6$-linked galactose branch unit). This suggests that mutants of pattern R-res-2 are unable to transfer galactose 
from UDPgalactose to form the $\alpha_{\mathrm{I}} \cdot 3$-linked unit, the disability demonstrated by assay in vitro in rfaH mutants (Osborn, I968). But broken cell preparations of strain TVI 48 have normal activity of this enzyme (M. J. Osborn, personal communication) and TVI48 is resistant to phage $\mathrm{C}_{2} \mathrm{r}$ to which mutants lacking galactose transferase are sensitive. The surmise that mutants of pattern R-res-2 are deficient in formation of the distal glucose unit (i.e. are of class $r f a J$ ) is thus disproven by the LPS structural studies. As the nature of their enzyme defect is unclear, the locus affected in such mutants may be termed $r f a$ (R-res-2). Both $r f a$ (R-res-I) and $r f a$ (R-res-2) map between cysE and pyrE (Kuo \& Stocker, 1968).

Many of the phage-resistant mutants which we isolated resembled the class $\mathrm{D}$ part-rough mutant St/22 of Yamamoto \& Anderson (I96I) both in cultural and serological properties and in phage sensitivity. The reactions of the newly isolated class D mutants to phages 6SR and $\mathrm{Br} 2$ divided them into three classes, termed D-I, D-2 and D-3 (Table 3). The affected loci were close to $x y l$, in some mutants at least between $x y l$ and $p y r E$ (Table 3). Sanderson $\&$ Saeed (1968) showed that the $r f$. loci of some class-D part-rough mutants are co-transducible with cysE and with pyrE. The cultural and serological properties and the partial sensitivity to smooth-specific phages (including susceptibility to transduction by $\mathrm{P}_{22}$ and/or P22h, (Gemski \& Stocker, 1967)) of mutants giving patterns D-I, D-2, and D-3 suggest that their LPS contains some O-specific material; and the LPS from all class D mutants so far analysed have been found to contain O-specific sugars (Naide et al. 1965; Hellerqvist \& Lindberg, I97I; Table 6). At least some class D mutants accumulate O-specific hapten (Table 6), a property characteristic of $r f a$ mutants. It is therefore plausible to infer that the three class D phenotypes (patterns D-I, D-2 and D-3) all result from mutation of $r f a$ genes causing LPS core defects which impede, but do not entirely prevent, the addition of $\mathrm{O}$-specific chains to the core. Two kinds of defect might have this effect: (i) an incomplete ('leaky') deficiency of a glycosyl transferase for addition of any of the sugars of the core side-chain if only the minority of core side-chains which are completed can accept $O$ chains (Naide et al. 1965); (ii) a complete defect of a glycosyl transferase for attachment of the $N$-acetylglucosamine side-branch of the core side-chain (or of some other, unidentified, side-branch), if the absence of such a branch reduces but does not abolish the ability of the core to accept $O$ side-chains. The nature of the defects and the identity of the loci concerned in mutants of patterns D-I, D-2 and D-3 are as yet unclear. Methylation studies on rough LPS extracted from the originally-described class D mutant, SL733 (=st/22), a mutant of pattern D-I, indicate that its rough core side-chains (i.e. those uncapped by $\mathrm{O}$ chains) lack the acetylglucosamine side-branch, some of them lacking also the $\alpha \mathrm{I} \cdot 6$-linked galactose side-branch (Hellerqvist \& Lindberg, I97I). This suggests that mutants of pattern D-I are deficient in a transferase for addition of the acetylglucosamine unit, and are therefore of class $r f a K$ in the scheme of Mäkelä \& Stocker (1969). It may be, as Hellerqvist \& Lindberg (197I) propose, that LPS core lacking acetylglucosamine can accept $O$ chains in vivo to a very limited extent, so that even a complete defect of this transferase would result in production of LPS containing some O-specific material, i.e. of the class D part-rough phenotype. However, it also seems possible that SL733 (and/or other mutants of pattern D-I) have a leaky defect in the addition of acetylglucosamine and that only the few core side-chains which receive this unit are then capped by addition of $O$ chains. The defects in mutants giving patterns D-2 and D-3 remain to be determined. The RI serological activity of LPS from two of the three class D-2 mutants tested by Nakane \& Nikaido (Table 6) suggest that these mutants have leaky defects in the reaction blocked in mutants of pattern R-res- $I$, whose LPS is of type RI. Until their biochemical defects are more precisely determined it may be convenient to refer to the loci affected in the three classes as $r f a(\mathrm{D}-\mathrm{I}), r f a(\mathrm{D}-2)$ and $r f a(\mathrm{D}-3)$. 
Mutants lacking transferases for the addition of the glucose no. I or of the galactose no. I unit, termed respectively $r f a G$ and $r f a H$ mutants, were detected by their distinctive patterns of phage sensitivity, including sensitivity to phage $\mathrm{C}_{2} \mathrm{I}$; all such mutants tested resulted from mutation in or near the strA-xyl-metA region (Osborn, 1968; Wilkinson \& Stocker, I968).

One of four mutants whose growth was inhibited by bile-salts made LPS deficient in the proximal heptose unit (class $r f a E$ ) and two at least made LPS deficient in the distal heptose unit (class $r f a F$ ); all four mutations mapped in or near the $\operatorname{str} A-x y l$-met $A$ region. The symbol $r f a E$ has been proposed for mutants deficient in the addition of the proximal heptose unit and $r f a F$ for those deficient in formation of the distal heptose unit (Mäkelä \& Stocker, I969). Note that this usage does not imply that the affected loci specify corresponding heptosyl transferases, since the defects may be in biosynthesis of the unknown precursor or precursors of the heptose units of the LPS core, which may be of two different types (Adams, Quadling \& Perry, 1967). Uncommon nucleotides have been detected in cell extracts of some of these mutants: UDPrhamnose in the $r f a E$ mutant SLIIO2 (Ginsburg, I966) and ADPmannitol in the rfaF mutants SLI I8I and SLI I82 (Scher \& Ginsburg, I968). It is not known if the presence of these nucleotides results from the biochemical defects responsible for the LPS anomalies of these strains.

If our attribution of class $\mathrm{D}$ part-rough phenotypes to defects, perhaps leaky, in biosynthesis of the LPS core is correct then all the mutations affecting only this function which have been mapped fall in or near the $\operatorname{str} A-x y l$-metA region; and if our his-linked culturally smooth mutants are, as we suppose, leaky $r f b$ mutants then all our mapped mutants with defects only in biosynthesis of the $\mathrm{O}$ repeat unit (of group B) resulted from mutation near his. By contrast genes for enzymes used both in LPS biosynthesis and in carbohydrate catabolism, namely phosphomannoisomerase and UDPgalactose epimerase, map elsewhere than these two regions (as also does $r f c$, for polymerization of $\mathrm{O}$ units). For a discussion of the significance of clustering of LPS genes see Mäkelä \& Stocker (1969).

Two classes of mutant, the hapten-positive $r f b T$ mutants and the $r f a L$ mutants, both of phage pattern R-sens, synthesize complete core LPS and accumulate $O$ hapten, and absence of partial deficiency of enzymatic activity for transfer of $O$ chains from antigen carrier lipid to LPS core has been demonstrated in vitro in at least one representative of each class (Cynkin \& Osborn, 1968; M. J. Osborn, personal communication). Perhaps these two loci, one near his, the other in the $r f a$ region, specify two enzymes, both involved in the transfer of O-chains, or perhaps one enzyme made up of two different polypeptide chains. Presumably at least part of an enzyme concerned in transfer of $O$ chains must recognize the sugar(s) at the reducing end of the chain, where it is joined to the antigen carrier lipid. Perhaps $r f b T$, in or near the main $r f b$ cluster, specifies a polypeptide (enzyme or enzyme component) specific for a given kind of $\mathrm{O}$ repeat unit. It seems likely that $r f a L$, remote from the $r f b$ cluster, specifies an enzyme or enzyme component which is indifferent to the character of the $\mathrm{O}$ repeat unit, since recombinants deriving the his-rfb region of their chromosome from a parent of a different $O$ group are able to transfer O units to LPS core (Mäkelä, 1966).

Our conclusions about the LPS character of mutants of different phage sensitivity patterns permit some inferences about the specificities of the phages used. Strain LT2 is lysogenic for phage Fels 2 (Yamamoto, 1967), but the presence or absence of this phage in our LT2 mutants did not qualitatively affect their sensitivity to any of the phages used in our pattern tests (though lysogeny for Fels 2 protects against the LPS-indifferent phage ESI 8 (Kuo \& Stocker, 1970)). Lysogeny for $\mathrm{P}_{22}$ protected smooth strains against $\mathrm{P}_{22}$ and $\mathrm{P}_{22} \mathrm{~h}$ but not against the other smooth-specific phage used (9NA), and protected non-smooth mutants against the rough-specific phage P22I. With these exceptions we assume that the sensitivity 
or resistance of a given LT2 derivative indicates its ability or inability to adsorb the phage concerned. (For direct evidence on correlation of phage sensitivity with affinity of phage for LPS see Lindberg \& Hellerqvist, 1971.) It should be noted that we recorded only sensitivity or resistance as determined by application of phage of an arbitrarily chosen concentration, usually on only a single test medium. A record of sensitivity therefore does not necessarily indicate high efficiency of plating, and strains recorded as resistant might have been scored as sensitive on some other medium, or if higher concentrations of phage had been used.

Our observations indicate that phages $\mathrm{P}_{22}, \mathrm{P}_{22} \mathrm{~h}$ and $9 \mathrm{NA}$ attack LT2 strains only if their LPS contains a certain proportion of O-specific side-chains, made of more than one repeating unit each (since $r f c$ mutants, which have single O-unit side-chains, were resistant to these phages). Measurement of the rate of adsorption of phage P22 by mutants with various LPS defects showed good correlation of adsorption rate with abundance of O-specific sugar in LPS (Lindberg, Sarvas \& Mäkelä, 1970). Their susceptibility as recipients in transduction with phages P22 and P22h (Gemski \& Stocker, 1967) indicates that most of our class D (= leaky $r f a$ ?) mutants and culturally smooth (leaky?) $r f b$ mutants retain some ability to adsorb these phages, even though they may appear resistant to them as ordinarily tested.

Lindberg and his colleagues (Lindberg, 1967; Lindberg \& Holme, 1969) conclude that the $N$-acetylglucosamine side-branch of the LPS core side-chain constitutes an essential part of the receptor site for Felix $\mathrm{O}$ phage, which is active on all non-smooth strains which make complete LPS core, on $r f c$ mutants and on smooth strains. All our observations are compatible with this conclusion, except that we observed partial, variable sensitivity to this phage with two $r f a H$ mutants (Wilkinson \& Stocker, I968), which might, however, be a consequence of leakiness of their $r f a$ defects. Phages Ffm and Br6o seem to be rather non-specific, in that they attacked nearly all non-leaky, and most leaky, rough mutants but did not attack smooth or $r f c$ mutants. Presumably the presence of even a single $O$ repeating unit attached to the core side-chain masks the sites on the core for which these phages have affinity. Phage 6SR in our experiments attacked only (i) rough mutants with complete core LPS (i.e. classes $r f b, p m i$ and $r f a L$ ) and (ii) one $r f a$ class, D-I, probably lacking the acetylglucosamine of the core side-chain. However, in subsequent experiments (Table 3; Stocker, 1969; B. A. D. Stocker, unpublished observations; A. Lindberg, personal communication) phage 6SR has given plaques on many $r f a$ mutants with deeper defects, including even $r f a E$. Variation in susceptibility to this phage may result from uncontrolled variation in divalent cation content of the nutrient agar used (A. Lindberg, personal communication; see also Kay, 1955, 1956).

Phage $\mathrm{C} 2 \mathrm{I}$ does not lyse Escherichia coli $\mathrm{K}$ I 2 wild-type (in respect of galactose metabolism) but does attack its mutants unable either to make UDPglucose, i.e. class galU, lacking UDPglucose pyrophosphorylase, or UDPgalactose, i.e. class galE, lacking UDPgalactose epimerase (Shedlovsky \& Brenner, 1963). Correspondingly in our experiments C2I attacked Salmonella typhimurium mutants unable to add the proximal glucose unit of the LPS core through lack of a glycosyl transferase (class $r f a G$ ) and also those unable to add the mainchain, $\alpha \mathrm{I} \cdot 3$-linked galactose unit which could result either from inability to make UDPgalactose ( $\mathrm{galE}$ ) or from deficient galactosyl transferase activity $(\mathrm{rfaH})$. Phage $\mathrm{C} 2 \mathrm{I}$ did not, however, attack strain TVI48 (nor other mutants of pattern R-res-2), even though according to analyses using the methylation method the core side-chains of TVI48 LPS lack the mainchain galactose unit (Hellerqvist \& Lindberg, 197I). The nature of the C2I adsorption site cannot be inferred from these results.

Phage $\mathrm{Br} 2$ attacks mutants making complete core LPS but apparently has some specificity as to the core structures required for its adsorption, since it attacks $\mathrm{rfaH}$ mutants, whose 
LPS lacks the main-chain galactose unit, but does not attack $r f a G$ mutants, lacking the proximal glucose unit and also attacks $r f a$ (R-res-I) mutants but not $r f a$ (R-res-2) mutants, classes whose LPS defects, discussed above, are certainly different. We cannot infer the nature of the chemical groups required for adsorption of this phage. Phage P22 I was active on all classes of non-smooth mutant, including $r f c$, but inactive on smooth strains. This may indicate that it is adsorbed not by any LPS structure but by some other component of the bacterial surface, and that the presence of numerous long $O$ side-chains on LPS hinders the access of this phage to its adsorption site.

Most of this paper is based on the University of London Ph.D. thesis (1966) of R. G. Wilkinson who was a United Kingdom Government Commonwealth Scholar; P. Gemski held a postdoctoral Fellowship from the National Institute of Allergy and Infectious Diseases, U.S. Public Health Service.

We are greatly indebted to our colleagues mentioned in the text for the supply of bacterial strains and phages and, especially, for permission to quote the unpublished results of their examination of our mutants.

\section{REFERENCES}

Adams, G. A., Quadling, C. \& Perry, M. B. (1967). D-glycero-D-manno heptose as a component of lipopolysaccharides of Gram-negative bacteria. Canadian Journal of Microbiology 13, I605-1613.

Beckmann, I., Subbaiah, T. V. \& Stocker, B. A. D. (1964). Rough mutants of Salmonella typhimurium. (2). Serological and chemical investigations. Nature, London 201, I299-I 30I.

Boyd, J. S. K. (1950). The symbiotic bacteriophages of Salmonella typhimurium. Journal of Pathology and Bacteriology 62, 501-517.

Boyd, J. S. K. \& Bidwell, D. E. (1957). The type A phages of Salmonella typhimurium: identification by a standardized cross immunity test. Journal of General Microbiology 16, 2 1 7-228.

BRANDIS, H. (1956). Untersuchungen über Bakteriophagen, die auf rauhe Bakterienstämme wirken. Zentralblatt für Bakteriologie, Parasitenkunde, Infektionskrankheiten und Hygiene, Abt. I, (Originale) 165, 305$3 \mathbf{I 2}$.

BraNDIS, H. (I966). Untersuchungen über die Wirkung von R-spezifischen Bakteriophagen auf R-Formen von S. paratyphi в. Zentralblatt für Bakteriologie, Parasitenkunde, Infektionskrankheiten und Hygiene, Abt. I, (Originale) I99, I85-192.

BRown, T. F. (1970). Characterization of bacteriophage $9 N A$. M.S. Thesis, Howard University, Washington, D.C.

BURNET, F. M. (1927). The relationships between heat stable agglutinogens and sensitivity to bacteriophage in the Salmonella group. British Journal of Experimental Pathology 8, 12 I-129.

BURNET, F. M. (1929). 'Smooth rough' variation in bacteria in its relation to bacteriophage. Journal of Pathology and Bacteriology 32, 15-42.

BURNET, F. M. \& MCKIE, M. (I933). The classification of dysentery-coli bacteriophages. I. The differentiation by Bail's methods of phages lysing a typical B. coli strain. Journal of Pathology and Bacteriology 36, 299-306.

CALLOW, B. R. (1959). A new phage-typing scheme for Salmonella typhi-murium. Journal of Hygiene, 57, 346-359.

CYNKIN, M. A. \& OsBorn, M. J. (1968). Enzymatic transfer of O-antigen to lipopolysaccharide. Federation Proceedings 27, 293.

Demerec, M., Adelberg, E. A., Clark, A. J. \& Hartman, P. E. (i966). A proposal for a uniform nomenclature in bacterial genetics. Genetics 54, $6 \mathrm{I}-76$.

Felix, A. \& Callow, B. R. (I943). Typing of paratyphoid B bacilli by means of Vi bacteriophage. British Medical Journal ii, $127-130$.

FiLDES, P. (1954). The relation of divalent metals to lysis of typhoid bacilli by bacteriophages. British Journal of Experimental Pathology 35, 122-1 28.

FILDES, P. \& KAY, D. (1955). The rate of adsorption of bacteriophage by rough and smooth strains of Salmonella typhi. British Journal of Experimental Pathology 36, 534-537.

Fukasawa, T. \& Nikaido, H. (1960). Formation of phage receptors induced by galactose in a galactosesensitive mutant of Salmonella. Virology 11, 508-510. 
Fukasawa, T. \& Nikaido, H. (I96I). Galactose mutants of Salmonella typhimurium. Genetics 46, I 295 I 303.

Gemski, P., Jun. \& Stocker, B. A. D. (1967). Transduction by bacteriophage P22 in nonsmooth mutants of Salmonella typhimurium. Journal of Bacteriology 93, I588-I 597.

GINSBURG, V. (I966). Isolation of uridine 5'-diphosphate L-rhamnose from Salmonella typhimurium. Journal of Biological Chemistry 24I, 3750-3753.

HÄMMERLING, G. (1968). Klassifizerung einiger Mutanten von Enterobacteriaceen. Diplomarbeit, University of Freiburg, Germany.

Hellerqvist, C. G. \& Lindberg, A. A. (197I). Structural studies on the common core polysaccharide from Salmonella typhimurium. Carbohydrate Research $16,39-48$.

JoYs, T. M. \& STOCKeR, B. A. D. (1965). Complementation of non-flagellate Salmonella mutants. Journal of General Microbiology 41, 47-55.

Kallings, L. O. (1967) Sensitivity of various Salmonella strains to Felix O-I phage. Acta pathologica et microbiologica scandinavica 70, 446-460.

KAY, D. (1955). The reactions of certain bacteriophages with their receptors isolated from Salmonella typhi O9OIR. British Journal of Experimental Pathology 36, 290-297.

KAY, D. (1956). The effect of divalent metals on the stability of a typhoid bacteriophage and its reaction with its receptor. British Journal of Experimental Pathology 37, 560-565.

KAY, D. (1962). The nucleic acid composition of bacteriophage $\phi \mathrm{R}$. Journal of General Microbiology 27 , 20I-207.

Kuo, T. \& STocker, B. A. D. (1968) Transduction of rta (= rouA) genes of Salmonella typhimurium strain LT2 by phage ESi 8 . Bacteriological Proceedings, p. 56.

Kuo, T. \& Stocker, B. A. D. (1970). ESı8, a general transducing phage for smooth and nonsmooth Salmonella typhimurium. Virology 42, $62 \mathrm{I}-632$.

LEDERBERG, J. (1950). Isolation and characterization of biochemical mutants of bacteria. Methods in Medical Research 3, 5-22.

LindBerg, A. A. (1967). Studies of a receptor for Felix O-I phage in Salmonella minnesota. Journal of General Microbiology 48, 225-233.

Lindberg, A. A. \& Hellerovist, C. G. (I97I). Bacteriophage attachment sites, serological specificity and chemical composition of the lipopolysaccharides of semirough and rough mutants of Salmonella typhimurium. Journal of Bacteriology 105, 57-64.

LindBerg, A. A. \& Holme, T. (1968). Immunochemical studies on cell-wall polysaccharide of rough mutants of Salmonella typhimurium. Journal of General Microbiology 52, 55-65.

Lindberg, A. A. \& Holme, T. (1969). Influence of O side chains on the attachment of the Felix O-I bacteriophage to Salmonella bacteria. Journal of Bacteriology 99, 5I3-5I9.

Lindberg, A. A., Sarvas, M. \& Mäkelä, P. H. (1970). Bacteriophage attachment to the somatic antigen of Salmonella: effect of O-specific structures in leaky $\mathrm{R}$ mutants and $\mathrm{S}$, TI hybrids. Infection and Immunity $\mathbf{I}$, 88-97.

Loveless, A. \& Howarth, S. (1959). Mutation of bacteria at high levels of survival by ethyl methane sulphonate. Nature, London r84, $1780-1782$.

Lüderitz, O., Galanos, C., Risse, H. J., Ruschmann, E., Schlecht, S., Schmidt, G., Schulteholthausen, H., Wheat, R., WestPhal, O. \& SChlosshardt, J. (I966). Structural relationships of Salmonella O and $\mathrm{R}$ antigens. Annals of the New York Academy of Sciences 133, 349-374.

LÜDERITZ, O., JANN, K., \& WHEAT, R. (I968). Somatic and capsular antigens of Gram-negative bacteria. In Comprehensive Biochemistry 26A, pp. 105-228. Edited by M. Florkin and E. H. Stotz. New York: Elsevier Publishing Co.

MÄKELÄ, P. H. (1966). Genetic determination of the O-antigens of Salmonella groups B (4, 5, I2) and C $(6,7)$. Journal of Bacteriology 91, I I I 5-I I 25 .

Mäkelä, P. H. \& Stocker, B. A. D. (1969). Genetics of polysaccharide biosynthesis. Annual Review of Genetics 3, 29I-322.

Markovitz, A., Sydiskis, R. J. \& Lieberman, M. M. (I967). Genetic and biochemical studies on mannosenegative mutants that are deficient in phosphomannose isomerase in Escherichia coli K -12. Journal of Bacteriology 94, I492-I496.

Naide, Y., Nikaido, H., Mäkelä, P. H., Wilkinson, R. G. \& Stocker, B. A. D. (1965). Semirough strains of Salmonella. Proceedings of the National Academy of Sciences of the United States of America 53, I47-I 53.

Nikaido, H., NAIDE, Y. \& MäKelä, P. H. (I966). Biosynthesis of O-antigenic polysaccharides in Salmonella. Annals of the New York Academy of Sciences 133, 299-314. 
Nikaido, H., Nikaido, K., Subbaiah, T. V. \& Stocker, B. A. D. (1964). Rough mutants of Salmonella typhimurium. (3) Enzymatic synthesis of nucleotide-sugar compounds. Nature, London 201, 1301-1 302.

OsBorN, M. J. (1968). Biochemical characterization of mutants of Salmonella typhimurium lacking glucosyl and galactosyl lipopolysaccharide transferases. Nature, London 217, 957-960.

Risse, H. J., Droge, W., Ruschmann, E., LüderitZ, O., WestPhal, O. \& SchlosshardT, J. ( I967). Eine neue Gruppe von Salmonella R-Mutanten: Serologische und biochemische Analyse des Heptosekerns von Lipopolysacchariden aus Salmonella minnesota- und Salmonella ruiru-Mutanten. European Journal of Biochemistry 1, 21 6-232.

Rosen, S. M., Zeleznick, L. D., Fraenkel, D., Wiener, I. M., Osborn, M. J. \& Horecker, B. L. (I965). Characterization of the cell wall lipopolysaccharide of a mutant of Salmonella typhimurium lacking phosphomannose isomerase. Biochemische Zeitschrift 342, 375-386.

SANDERSON, K. E. (1970). Current linkage map of Salmonella typhimurium. Bacteriological Reviews 34, 176-193.

Sanderson, K. E. \& Hall, C. A. (1970). F-prime factors of S. typhimurium and an inversion between S. typhimurium and E. coli. Genetics 64, 215-228.

SANDERson, K. E. \& SAEED, Y. A. (1968). Genetics of cell wall mutants of Salmonella typhimurium. Bacteriological Proceedings, 55, 80.

SCHER, B. M. \& GinsBuRG, V. (1968). Isolation of adenosine 5'-diphosphate D-mannitol from Salmonella typhimurium. Journal of Biological Chemistry 243, 2385-2389.

SCHMIDT, G. \& LÜDERITZ, O. (1969). Untersuchungen zur Typisierung von Salmonella-R-Formen. 2. Typisierung von S. minnesota-Mutanten durch Phagen. Zentralblatt für Bakteriologie, Parasitenkunde, Infektionskrankheiten und Hygiene, Abt. I, (Originale) 210, 381-387.

Sertic, V. \& Boulgakov, N. (1935). Classification et identification des typhi-phages. Comptes rendu des séances de la Société de biologie Ir9, I270-1272.

ShedLOVSKY, A. \& BRENNER, S. (1963). A chemical basis for the host induced modification of T-even bacteriophages. Proceedings of the National Academy of Sciences of the United States of America 50, 300-305.

SinsheImer, R. L. (1959). A single-stranded deoxyribonucleic acid from bacteriophage $\phi$ XI74. Journal of Molecular Biology I, 43-53.

SмrтH, S. M. \& Stocker, B. A. D. (1962).Colicinogeny and recombination. British Medical Bulletin 18, 46-5I.

SPICER, C. C. \& DATTA, N. (I959). Reversion of transduced antigenic characters in Salmonella typhimurium. Journal of General Microbiology 20, 136-143.

STOCKER, B. A. D. (1969). Genetic determination of the structure of the core of Salmonella somatic polysaccharide. Colloques Internationaux du centre National de la Recherche Scientifique 174, 189-199.

Subbaiah, T. V. \& Stocker, B. A. D. (1964). Rough mutants of Salmonella typhimurium. (I) Genetics. Nature, London 201, 1298-I 299.

TAYLOR, A. L. (1970). Current linkage map of Escherichia coli. Bacteriological Reviews 34, 155-175.

TesSman, I. (1959). Some unusual properties of the nucleic acid in bacteriophages $S_{1} 3$ and $\phi X_{174}$. Virology 7 , 263-275.

Tessman, E. S. \& Shleser, R. (1963). Genetic recombination between phages $\mathrm{Si}_{3}$ and $\phi \mathrm{X}_{1} 74$. Virology 19. 239-240.

TUCKER, R. G. (196r). The role of magnesium ions in the growth of Salmonella phage anti-R. Journal of General Microbiology 26, 313-323.

Wilkinson, R. G. \& StockeR, B. A. D. (1968). Genetics and cultural properties of mutants of Salmonella typhimurium lacking glucosyl and galactosyl lipopolysaccharide transferases. Nature, London 217, 955-957.

Yамамото, N. (1967). The origin of bacteriophage P221. Virology 33, 545-547.

YAMAMoto, N. \& ANDERSON, T. F. (1961). Genomic masking and recombination between serologically unrelated phages $\mathrm{P} 22$ and P221. Virology 14, 430-439.

Yamamoto, N. \& Weir, M. L. (1966). Boundary structure between homologous and non-homologous regions in serologically unrelated bacteriophages P22 and P22I. I. Mapping by recombination found in cultures of double lysogenic strains for P22h and P22I. Virology 28, 325-330.

Yuasa, R., NAKANe, K. \& Nikaido, H. (I970). Structure of lipopolysacharide from a semirough mutant. European Journal of Biochemistry 15, 63-7I.

ZAHLER, S. A. (1958). Some biological properties of bacteriophages $S_{13}$ and $\phi X-174$. Journal of Bacteriology 75, 310-315.

ZINDER, N. D. (1953). Infective heredity in bacteria. Cold Spring Harbor Symposia on Quantitative Biology 18, 26I-269.

ZINDER, N. D. (1958). Lysogenisation and superinfection immunity in Salmonella. Virology 5, 29I-326. 